\title{
Nanoscopic investigation of C9orf72 poly-GA oligomers on nuclear membrane disruption by photoinducible platform
}

\section{Hung-Ming Chien}

Institute of Chemistry, Academia Sinica

Ruei-Yu He

Institute of Chemistry, Academia Sinica

Chi-Chang Lee

Institute of Chemistry, Academia Sinica

Yung-An Huang

Institute of Chemistry, Academia Sinica

I-Ju Hung

Institute of Chemistry, Academia Sinica

Kai-Ting Hou

Institute of Chemistry, Academia Sinica

Jye-Chian Hsiao

Institute of Chemistry, Academia Sinica

\section{Po-Chao Lu}

Institute of Chemistry, Academia Sinica

Diksha Agnihotri

National Taiwan University

Eric Hwang

National Chiao Tung University

Jen-Tse Huang ( $\nabla$ jthuang@gate.sinica.edu.tw )

Institute of Chemistry, Academia Sinica https://orcid.org/0000-0003-4847-7126

\section{Article}

Keywords: nanostructures, amyotrophic lateral sclerosis (ALS), pathological roles

Posted Date: February 15th, 2021

DOI: https://doi.org/10.21203/rs.3.rs-209180/v1 
License: (c) (i) This work is licensed under a Creative Commons Attribution 4.0 International License. Read Full License

Version of Record: A version of this preprint was published at Communications Chemistry on July 23rd, 2021. See the published version at https://doi.org/10.1038/s42004-021-00547-6. 


\section{Abstract}

Dipeptide repeats (DPRs) translated from the mutated $C 9$ orf72 gene have recently been correlated with amyotrophic lateral sclerosis (ALS). Within these DPRs, the most abundant glycine-alanine (GA) DPRs form insoluble inclusions in C9orf72-ALS patients. While GA DPRs aggregates have been considered as amyloid, the biophysical features and cytotoxicity of GA DPRs oligomers generated during the amyloidogenesis has not yet been explored due to its unstable and fast equilibrium nature. In this study, we develop a photoinducible platform based on methoxynitrobenzene chemistry to enrich GA DPRs that allows to monitor the oligomerization process of GA DPRs in cells in nanoscale. By combining lifetimebased and super-resolution fluorescence microscopies with biophysical tools, we thoroughly examined the GA DPRs oligomerization process nanoscopically in a time-dependent manner. We provide direct ex vivo and in vitro evidences to demonstrate GA DPRs oligomers rather than nanofibrils disrupt nuclear membrane integrity. In addition, we found GA DPRs sabotage Ran protein gradient, hamper nucleocytoplasmic shuttling in neurons, and cause the mislocalization of TAR DNA-binding protein 43 in primary cortical neurons. Our results highlight the nanoscopic properties and toxicity of GA DPRs oligomers in living cells, which is a key step toward elucidating the pathological roles of C9orf72 DPRs in disease.

\section{Introduction}

$\mathrm{G}_{4} \mathrm{C}_{2}$ hexanucleotide repeat expansion in the noncoding region of the chromosome 9 open reading frame 72 (C9orf72) gene has been considered as the most prevalent genetic cause of amyotrophic lateral sclerosis (ALS) and frontotemporal dementia (FTD) recently. ${ }^{1-3}$ Until now, three distinct mechanisms have been deduced ${ }^{4-6}$ to explain how $\mathrm{G}_{4} \mathrm{C}_{2}$ hexanucleotide expansion mutation confers neurotoxicity: (1) Reduced C9orf72 gene products due to impaired transcription or splicing ${ }^{7-9}$; (2) G-quadruplex structure composed of the mutant RNA transcript to sequester essential RNA binding proteins ${ }^{10,11}$; and (3) the unconventional dipeptide repeats (DPRs) translated from the sense and antisense strands of the RNA transcript. ${ }^{12-17}$ While these proposed pathomechanisms are not mutually exclusive, these DPRs, namely glycine-alanine (GA), glycine-arginine (GR), glycine-proline (GP), proline-alanine (PA), and proline-arginine (PR) have been respectively identified in ALS or FTD patients with C9orf72 repeat expansions and thus drawn enormous attention. ${ }^{18,19}$

Among these DPRs, GA DPRs were the most abundant DPRs found in the C9orf72-mediated ALS patients ${ }^{20,21}$ and formed ubiquitin- and p62-positive inclusions in the cerebellum, hippocampus, and frontal cortex. ${ }^{22,23}$ Although many DPRs have been proposed to participate in amyloid-like cascade in the disease progression, ${ }^{24}$ only GA DPRs aggregates adopted beta-sheet conformation. ${ }^{25}$ Revealing by another cryo-electron tomography study, GA DPRs inclusions are composed of amyloid-like ribbons ${ }^{25}$ and exhibited pervasive sequestration of proteasome. ${ }^{12,14,26}$ Moreover, GA DPRs inclusions are also the perpetrator to blame for endoplasmic reticulum stress and synaptic-related proteins sequestration. ${ }^{26}, 27$ 
Collectively, these evidences suggested GA DPRs as a member of a growing list of misfolding agents in neurodegenerative diseases.

In last two decades, the oligomeric species of misfolding proteins have emerged as a driver of neurotoxicity. ${ }^{28}$ Despite of its aggregation-prone propensity and ribbon-like conformation, the existence of GA DPRs oligomers during disease progression remains unknown. To recapitulate the accumulation of DPRs within cells, the ectopic gene overexpression was routinely practiced through AUG-mediated translation. ${ }^{14,18,29,30}$ However, the bulky ectopic expression of GA DPRs may overshadow putative GA DPRs oligomers because the oligomeric intermediates would rapidly polymerize into mature fibrils. In addition, the instable expressivities of ectopically expressed products was also reported ${ }^{17}$ and extra tagging of reporters (e.g. GFP) may interfere with the intrinsic properties of GA DPRs. ${ }^{30}$ Therefore, a controllable platform that provide suitable window to observe GA DPRs oligomers in detail and characterize their proteinopathy in neurons is imperative.

To address the aforementioned questions, we have created photoinducible probes consisting of a cellpenetrating sequence (octalysine, $\mathrm{K}_{8}$ ), a GA DPRs [poly-(glycine-alanine), (GA) ${ }_{12}$ ] segment, a photolabile methoxynitrobenzene linker in the bridge (photolinker, $\mathrm{PL}$ ), and a cysteine at $\mathrm{N}$-terminus (ADP-1 in Figure $1 \mathrm{~A})$. Through a Michael addition reaction with photostable fluorophore (Alexa Fluor ${ }^{\mathrm{TM}} 488 \mathrm{C} 5$ Maleimide), we further prepared fluorescent probes for visualization (ADP-2 in Figure 1A). This newly-designed probe can easily penetrate cell membranes, prevent GA DPRs aggregation until photoinitiation, and induce GA DPRs oligomerization and fibrillization in neurons after photolysis. In comparison, we also synthesized probes with shorter repeats length GA DPRs $\left[(\mathrm{GA})_{3}\right]$ which could not oligomerize after photolysis as control (ADP-3 and ADP-4 in Figure S1). The repeat number of GA dipeptide in photoinducible probes was determined by the fact that $(\mathrm{GA})_{12}$ but not $(\mathrm{GA})_{3}$ formed amyloid nanofibrils (Figure S1-S2). By exploiting this minimalist platform, we characterized in-depth GA DPRs oligomerization and fibrilization process with both in vitro and ex vivo models in nanoscale. Accordingly, we correlated the susceptibility of nuclear membrane disruption and nuclear transport defect to the GA oligomers with its putative neurotoxicity.

\section{Results And Discussion}

\section{Photoinduced GA DPRs promptly form oligomers and subsequently turn into nanofibrils}

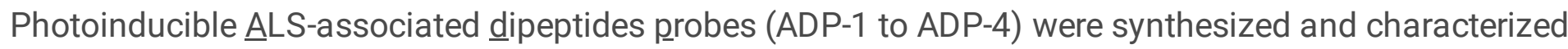
as described in Methods Section (Figure 1A, Figure S3-S6, and Table S1). To ensure ADP-1, [cysteine(glycine-alanine) ${ }_{12}$-photolinker-lysine 8 ], could undergo the photolysis reaction as shown in Figure $1 \mathrm{~A}$, we irradiated ADP-1 (50 $\mu \mathrm{M})$ solution with 365-nm light (power density: $32 \mathrm{~mW} / \mathrm{cm}^{2}$; duration: $1 \mathrm{~min}$ ) and then subjected to RP-HPLC and mass spectroscopy analyses (details in Supporting Information). Notably, two anticipated peptide fragments, respectively octalysine $\left(\mathrm{K}_{8}\right)$-attached with photolinker (retention time $\sim 14.5$ minute) and GA DPRs [(GA) $\left.{ }_{12}\right]$ (retention time $\sim 23$ minute), were separated and identified (Figure S3). 
After confirming the existence of the photoinduced GA DPRs, we next attempt to characterize their amyloid properties. Through the micrograph by transmission electron microscopy (TEM), we noticed liberated GA DPRs fragments promptly form spheroid aggregates in nanoscale at 2-hour-incubation time and give rise to entangled nanofibrils (with the width around $10 \mathrm{~nm}$ ) after 6 hours while unirradiated ADP1 form trace amorphous aggregates (Figure 1B and Figure S7). Moreover, we have determined the hydrodynamic diameter of the spheroids aggregates with dynamic light scattering (DLS) and found the mean diameter ( $83 \mathrm{~nm} \pm 19 \mathrm{~nm}$ ) (Figure S8) is compatible with that observed in TEM ( $28 \mathrm{~nm} \pm 8 \mathrm{~nm})$. The hydrodynamic diameter measured by DLS better reflects the size of spheroids aggregates rather than the diameter found by TEM which is proceed in dehydrated and near-vacuum environment. ${ }^{31}$ On the basis of the amyloidogenic properties of GA DPRs, we surmised the spheroid aggregates at 2 hours could be amyloid oligomers (Figure 1B) and thus immunoblotted the ADP-1-derived GA DPRs with A11, an amyloid oligomers-specific antibody, ${ }^{32}$ in a dot blot analysis. Interestingly, A11-reactivity of the GA DPRs reached the strongest after 2 hours incubation but soon receded as the incubation time extended, suggesting these transient spheroid aggregates are GA DPRs oligomers (Figure 1C).

In addition to oligomerization, the rapid accumulation of the $\beta$-sheet content of the photoinitiated ADP-1 over time in circular dichrosim (CD) as well as the enhanced fluorescence kinetic in thioflavin T (ThT) binding assay indicated the resultant GA DPRs underwent amyloidogenesis process (Figure S9). Moreover, in a time-course turbidity assay, the irradiated ADP-1 peaked within 48 hours and retained high readouts through the observation window while the unirradiated ADP-1 remained low turbidity (Figure 1D). In comparison, we have prepared another probe, ADP-3 [cysteine-(glycine-alanine) ${ }_{3}$-photolinkerlysine $_{8}$ ] as a control. As observed by TEM, both the irradiated and unirradiated ADP-3 failed to grow nanofibrils and showed no significant turbidity increase even after 96 hours incubation (Figure S10). Collectively, we demonstrated that GA DPRs fragments from ADP-1 probe are able to form amyloid oligomers and gradually turn into ThT-positive amyloid nanofibrils after photoinitiation.

\section{Visualizing GA DPRs oligomerization In vitro}

While we have detected GA DPRs oligomers and nanofibrils respectively in the photoinitiated ADP-1 as described above, we further exploited fluorescence lifetime imaging microscopy (FLIM) to monitor the aggregation process and elucidated their in-depth biophysical properties. As FLIM allows detailed monitoring the differences in fluorescence lifetime fluorophores in response to the local environment and interactions, it has been applied to trace the aggregation kinetic process of a fluorophore-attached proteins on the basis of fluorescence self-quenching property. ${ }^{33}$ While the fluorescence lifetime dropping, it is manifesting that these proteins or peptides would form highly compact structure. We thus decided to delineate the structural features of GA DPRs during oligomerization by applying time-course FLIM on the fluorophore-labeled probe, ADP-2 [Alexa Fluor 488-cysteine-(glycine-alanine) ${ }_{12}$-photolinker-lysine ${ }_{8}$ ]. As shown in Figure $1 E$ (upper panel), the average lifetime $(\lambda)$ for monomeric AF-488-labeled GA DPRs was $4.07 \mathrm{~ns}$ after photolysis and 0.5 hour incubation at $37^{\circ} \mathrm{C}$. After 2 hours incubation, ADP-2 exhibited an average lifetime of $4.02 \mathrm{~ns}(\lambda)$ with a number of puncta in FLIM, where denote the oligomeric species 
(Figure 1E, middle panel). Moreover, after 24 hours of incubation, as FLIM of ADP-2 showed markedly patchy pattern and a much lower average lifetime (3.72 ns) along with a broadened distribution, hinting the formation of nanofibrils (Figure 1E, bottom panel). Additionally, as a control, we also measured the fluorescence lifetime of the fluorophore-labeled probe, ADP-4 [Alexa Fluor 488-cysteine-(glycine-alanine) $3^{-}$ photolinker-lysine 8 ], in which the GA DPRs is much shorter. As expected, the fluorescence lifetime of irradiated ADP-4 remained around 4.1 ns through whole time course (Figure S11). The decline of fluorescence lifetime in irradiated ADP-2 suggested that GA DPRs released from photoinitiated ADP-2 would form the higher ordered aggregates, including oligomers and nanofibrils, in a time-dependent manner.

In addition, we applied direct stochastic optical reconstruction microscopy (dSTORM), which enables image acquisition at a resolution beyond the diffraction limit, to visualize GA DPRs fibrillization process at a submicron scale (Figure 1F). After 12 hours-incubation, GA DPRs developed linear aggregates with an average length of 2-5 $\mu \mathrm{m}$. Moreover, huge clustered aggregates (ranging from 5 to $10 \mu \mathrm{m}$ ) were observed after 24 hours-incubation. dSTORM clearly revealed that huge aggregates are composed of delicate and intertwist fibrillar networks, which was comparable with foregoing TEM observation (Figure 1B). Together with FLIM measurement, we made used of the fluorophore-labeled GA DPRs fragments from ADP-2 probe to visualize oligomerization process and finally turn into nanofibrils upon photoinitiation in a time course.

\section{ADP-1 can penentrate cells and induce GA DPRs oligomerization upon photoinitation}

As the biophysical properties of ADP-2 have been delineated in vitro, we next aim to monitor the cellpenetration abilities as well as oligomerization and aggregation process of ADP-2 in neuronal cell (SHSY5Y). As shown in FLIM, fluorescent GA DPRs released from ADP-2 evenly distributed in cells after photoinitiation and 2 hours incubation, with the corresponding average lifetime around $4.2 \mathrm{~ns}$, suggesting GA DPRs may remain the monomeric status (Figure 2A). As ADP-2-treated cell was incubated to 12 hours, GA DPRs gradually assembled into submicro- and micron-sized puncta in the cytosol, with the lifetime decrease with uneven distribution (ranging from 3.5 to 4.2 ns; Figure $2 \mathrm{~A}$ ). After 24 hours incubation, GA DPRs developed highly compacted cytosolic inclusions, with the lifetime decreased further (2.5-4.1 ns; Figure 2A). To further identify amyloid oligomers during GA DPRs oligomerization process in neurons, we immunoblotted with A11 antibody in the lysates from ADP-1 treated SH-SY5Y cells with or without photoinitiation in a time-dependent manner (Figure 2B, details in Methods). The resultant A11 signal was first normalized to the internal control (GAPDH) and then compared with the signal at 0.5-hour-incubation (Figure $2 \mathrm{~B}$ ). As the $\mathrm{A} 11$ signals peaked from $6^{\text {th }}$ hour to $12^{\text {th }}$ hours after photoinitiation, intracellular GA DPRs amyloid oligomers in ADP-1 treated neurons is also transient, consistent to what we have observed in vitro (Figure $2 \mathrm{C}$ ). Instead, A11 signals in ADP-3 treated SH-SY5Y cell lysates remained mostly unchanged through the same incubation window (Figure S12), suggesting ADP-3 (with shorter GA DPRs) failed to induce GA DPRs oligomerization. In short, we have demonstrated photoinducible probes (ADP-1 and ADP-2) as a feasible platform to introduce cytosolic GA DPRs and initiate oligomerization in cells upon photoinitiation. 


\section{Photoinitiated GA DPRs impaired nucleocytoplasmic transport in human neuroblastoma cells.}

Since it has been shown that the impairment of nucleocytoplasmic transport plays critical roles in ALS, ${ }^{14,34,35}$ we thus wonder if photoinitiated GA DPRs contribute similar phenotypes. We first examined the effect of GA DPRs on crucial cellular factors governing nuclear transport, including Ras-related nuclear protein (Ran) and importin- $\beta$. As an abundant protein in nucleus, Ran drives nuclear transport via its concentration gradient between nucleus and cytoplasm. ${ }^{36}$ After ADP-2 treatment followed by photoinitiation (hereafter referred as "ADP photoinitiated"), we observed cytosolic retention of Ran protein accompanied by GA DPRs inclusions in SH-SY5Y cells (Figure 3A), as demonstrated in intensity profile (Figure 3B). By contrast, Ran resided mainly in the nucleus in all control experiments (including the untreated, ADP-2-treated only, and photoinitiation only groups) (Figure 3A-C, and S13A), suggesting that the cytosolic GA DPRs may induce the disruption of the Ran gradient, thus leading to considerable Ran mislocalization into cytoplasm. On the other hand, we also examined the effect of GA DPRs on importin$\beta$, a client protein of Ran. Due to its weak binding affinity with FG-repeat domain within nucleopore complex, importin- $\beta$ located mainly at perinuclear space. ${ }^{37}$ We observed most importin- $\beta$ escaped from the perinuclear region in the presence of photoinitiated GA DPRs. In comparison, all controls exhibited perinuclear importin- $\beta$ (Figure 3D-F, and S13B). Moreover, we notice that treating cells with the control peptide (ADP-3), which could not undergo oligomerization process, fail to initiate the mislocalization of the aforementioned transport factors (Figure S14). Taken together, our results showed ADP-2-derived GA DPRs interrupt nucleocytoplasmic transport regarding the mislocalization of Ran and importin- $\beta$.

In addition to mislocalization of transport factors, we thereafter suspected addressed that how bidirectional nucleocytoplasmic transport function was impaired by GA DPRs. Consequently, we established a cargo distribution evaluation system by overexpressing green fluorescence proteins fused with both nuclear localization sequence (NLS) and nuclear export signal (NES) (denoted as shuttling GFP or SGFP) in SH-SY5Y cells (Figure 3G) to investigate the nucleocytoplasmic transport function. As sGFP shuttle between nucleus and cytoplasm rapidly due to its NLS and NES, ${ }^{15}$ one could easily characterize the impairment of nuclear import and export by examining the subcellular distribution of sGFP in cells. To define the distribution of SGFP, we monitored the nuclear-to-cytoplasmic ratio following the equation ( $\mathrm{N}$ to $\mathrm{C}$ ratio $=$ fluorescence intensity of sGFP in nucleus / fluorescence intensity of sGFP in cytoplasm) through imaging analysis. It is expected the disruption of nuclear export will result in higher $\mathrm{N}$ to $\mathrm{C}$ ratio while the impairment of nuclear import will have lower ratio than control (DMSO). To validate this system, either nuclear import (Importazole, IPZ) or nuclear export inhibitor (Leptomycin B, LMB) was applied as reference groups. As expected, while LMB caused most sGFP retained in the nucleus ( $N$ to $C$ ratio $=2.2$ ), IPZ led to sGFP accumulation in the cytosol $(\mathrm{N}$ to $\mathrm{C}$ ratio $=0.6$; Figure $3 \mathrm{G}-\mathrm{H})$. Notably, most of the sGFP remain in the cytosol upon ADP-1 treatment and photoinitiation, with the N-to-C ratio (0.5) being similar to that in IPZ (0.6; Figure 3G-H), indicating the photoinitiated GA DPRs impaired nuclear import.

\section{GA DPRs oligomers can disrupt the nuclear membrane.}


Since the nuclear transport impairment accompanied with nuclear membrane disruption have been linked with several neurodegenerative diseases, ${ }^{38-40}$ we thus examine the nuclear membrane ultrastructure in the presence and absence of ADP-1 under TEM. The neurons treated with ADP-1 displayed malformed nuclear morphology with massive folding and invaginations after photoinitiation (Figure 4A). In particular, in higher magnification, the interspace of the nuclear membrane became blurred and uneven after ADP-1 treatment (+ADP-1 +UV), indicating GA DPRs devastate nuclear membrane integrity (indicated with yellow arrows). By contrast, majority of control cells (including the untreated, ADP-1treated only, and photoinitiation only groups) exhibited smooth and robust nuclear membrane structure, where the inner and outer nuclear membranes could be clearly identified in high magnification (Figure 4A). Furthermore, we also examined whether nuclear lamina, a meshwork structure beneath nuclear membrane and provides mechanical support, ${ }^{41}$ would be affected in ADP-1 treated cells. By monitoring immunostaining of lamin B1, a nuclear lamina marker, we noticed the nuclear lamina abnormal pattern (indicated by yellow arrows) accompanied with GA DPRs inclusions (green) in ADP-2 photoinitiated cells (+ADP-2 +UV), which was seldom observed in controls (-ADP-2 -UV in Figure 4B and +ADP-3 +UV in Figure S15). As shown in intensity profile (Figure 4C), lamin B1 distributed through the nucleoplasm rather than tethering beneath the nuclear membrane. By quantifying the percentage of cells with abnormal lamin B1 staining pattern, we did confirm that GA DPRs induced higher abnormal lamin B1 pattern (51\%) than control (23\%) (Figure 4D, details in Methods). Furthermore, through two-color dSTORM, we nanoscopically depicted inhomogeneous staining of laminB1 along with the photoinitiated perinuclear GA DPRs inclusions (green) in the presence of ADP-2 (+ADP-2 +UV) while the smooth nuclear lamina (white) was observed in control (-ADP-2 -UV) (Figure S16). These morphological evidences indicated neuronal cells show severe defects in nuclear periphery in response to GA DPRs.

Given the fact that the oligomers of misfolded protein readily impair membrane structure, ${ }^{42}$ we conjectured that GA DPRs oligomers formed during the oligomerization process might be responsible for nuclear membrane disruption. To scrutinize the putative impingement of amyloid oligomers onto nuclear membrane, we have pretreated SH-SY5Y cells with digitonin, a detergent that selectively permeabilizes plasma membrane but leaves the nuclear membrane intact, ${ }^{43}$ to preserve the nucleus for the subsequent GA DPRs oligomers and nanofibrils treatments (details in Methods). Since the intact nuclear membrane generally precludes the free passage of macromolecules like antibody, the GA DPRs-treated nuclei were then immunostained with lamin B1 antibody and the lamin B1 antibody penetrance was used to evaluate the corresponding membrane permeability (Figure S17). Different from the mock (upper row in Figure 5A) and nanofibril-treated groups (bottom row in Figure 5A), the oligomer-treated nuclei (middle row in Figure 5C) exhibited the pronounced positive staining of lamin B1, revealing the GA DPRs oligomers enhance the penetrance of nuclear membrane. Moreover, the ADP-3-treated group failed to induce significant lamin B1 staining, demonstrated that neither $(\mathrm{GA})_{3}$ DPRs nor octalysine fragments is capable of impairing nuclear membrane (Figure S18). In order to evaluate the impact of GA DPRs on the nuclear membrane integrity, we defined: antibody penetrance level (\%) = number of nuclei with Lamin B1-positive staining / total nuclei numbers. We showed that "oligomers-treated group" has higher antibody penetrance level (62\%) comparing to either the nanofibrils-treated group (9\%), mock group (14\%), or ADP-3-treated group (10.4\%) 
(details in Methods) (Figure 5B). These findings revealed that it could be the GA DPRs oligomers rather than the nanofibrils compromise the nuclear membrane integrity.

In order to clarify whether GA DPRs oligomers can disrupt lipid membranes, we monitored the fluorescence enhancement of calcein-encapsulated lipid-based liposomes in the presence and absence of different forms (either oligomers or nanofibrils) of GA DPRs. Since the fluorescence of calcein is selfquenched within the liposome but restored when calcein leaks out, ${ }^{44}$ we herein define the membrane leak out level (\%) as the following. Leak out $(\%)=[($ Enhanced fluorescence signal background signal) / fluorescence signal with Triton X100] (details in Methods). We found GA DPRs oligomers photoinduced by ADP-1 cause substantial release of liposomal content ( 33\%) within 2 hours, whereas GA DPRs nanofibrils have minimal effect ( $6 \%$ ) on calcein leakage (Figure $5 \mathrm{C})$. Notably, the control peptide, ADP-3, had negligible effect $(\sim 7 \%)$ on the encapsulated liposome. Collectively, we demonstrated the lipid membrane was disrupted mainly by GA DPRs oligomers rather than GA DPRs nanofibrils, short GA DPRs $\left[(\mathrm{GA})_{3}\right]$, or octalysine.

\section{GA DPRs induced endogenous TDP-43 mislocalization in human neuroblastoma}

In addition to DPRs, TAR DNA-binding protein 43 (TDP-43) proteinopathy is also considered as the hallmark in C9orf72-mediated ALS. ${ }^{45,46}$ Studies have reported the mislocalization of TDP-43 induced by GA DPRs inclusions in HeLa cells ${ }^{47}$ and the coaccumulation of phosphorylated TDP-43 and GA DPRs

proteins in neurons and diseased human brains. ${ }^{30,48}$ On the basis of previous findings, ${ }^{49-51}$ the association between GA DPRs inclusions and TDP-43 for C9orf72-mediated ALS pathogenesis are highly suspected, which drew our attention. In ADP-1-photoinitiated SH-SY5Y cells, through immunocytochemistry, we found most endogenous TDP-43 (green) translocated from the nucleus (blue) to the cytoplasm after photoinitiation (Top row in Figure 6A). In contrast, TDP-43 resided in nuclei in all control groups (Figure 6A-B, Figure S19). We thus reasoned endogenous TDP-43 mislocalization may result from the influence of GA DPRs-driven nuclear transport failure.

\section{Introduction of GA DPRs caused severe damage to mouse cortical neurons.}

Studies have demonstrated that expressing GA DPRs in mouse cortical tissue induced cognitive deficits along with cerebellar atrophy, neural toxicity, and astrogliosis. ${ }^{14,52}$ To examine the effect of GA DPRs on cortical neurons, ADP-1 was applied to dissociated primary cortical neurons which had been further cultured for 21 days in vitro (21DIV). This prolonged culturing time allowed cortical neurons to mature and establish synaptic connections between each other, ${ }^{53}$ effectively mimicking the physiological condition that occurs when C9orf72-mutation-mediated effects begin. Upon ADP-1 photoinitiation, the cortical neurons exhibited severe neurite fragmentation (through immunostaining with neuron-specific $\beta$ III-tubulin antibody TUJ1) and endogenous TDP-43 mislocolization (Figure 6C-D, see Methods). In contrast to ADP-1- photoinitiated neurons, all control groups showed elaborate neurite arborization and minimal neurite fragmentation (including the untreated, ADP-1-treated only, and photoinitiation only 
groups) (Figure 6C-D and Figure S20). We thus showed photoinitiated ADP-1 cause acute toxicity in primary cortical neurons, evincing GA DPRs induce cortical neuron degeneration.

\section{Conclusion}

In summary, we successfully created a photoinducible platform to deliver GA DPRs into neurons. While amyloid oligomerization from monomers to nanofibrils is a quick equilibrium process, our platform underscores the possible roles of GA DPRs oligomers in C9orf72-mediated ALS. Through biophysical measurements, we comprehensively monitored the GA DPRs nanostructure and the oligomerization and fibrillization process in vitro and in neuronal cells. In respect of the observed nuclear transport defects (e.g. Ran mislocalization and sGFP cytosolic accumulation), we reasoned that GA DPRs may induce nuclear membrane disruption. Along with ultrastructural studies, we confirmed GA DPRs perturbs the nuclear membrane by observing the invaginated nuclear membrane and abnormal distribution of nuclear lamina upon ADP-1 photoinitiation. We probed the presence of amyloid oligomers in cells through the A11 immunoblotting. Given the amyloid oligomers tend to interfere the lipid membrane, with further ex vivo and in vitro experiments, we have shown GA DPRs oligomers, rather than nanofibrils, disrupt the lipid membranes. Additionally, aberrant cytosolic accumulation of endogenous TDP-43 in neurons through our probes showed the possible correlation of GA DPRs with TDP-43 in disease. Conclusively, we confirmed the nuclear membrane disruptive ability of photoinitiated GA DPRs oligomers and thus provided new insight of the possible pathological roles of GA DPRs, which may ultimately benefit on the mechanistic studies of C9orf72-mediated ALS.

\section{Methods}

\section{Polypeptide synthesis.}

Both amino acids and 4-[4-[1-(9-fluorenylmethyloxycarbonylamino)ethyl]-2-methoxy-5-nitrophenoxy]-nbutanoic acid (Fmoc-photolabile linker) were purchased from Advanced ChemTech. All peptides were synthesized by the standard Fmoc polyamide chemistry on Rink Amide AM resin (200-400 mesh, MerckMillipore, Germany) using the automated peptide synthesizer Liberty Blue ${ }^{\mathrm{TM}}$ (CEM, U.S.A.). Peptides were cleaved from the resin with cleavage cocktail (90 \% TFA / $2.5 \%$ water / $2.5 \%$ TIPS / 5 \% EDT). Crude polypeptides were purified with high-performance liquid chromatography (HPLC) (1260 Infinity LC system, Agilent, U.S.A.) equipped with a C18 reverse-phase semi-preparative column (Shiseido, Japan). The gradient separation was achieved by mixing buffer A ( $5 \%$ acetonitrile / $0.1 \%$ TFA / $94.9 \%$ water) and buffer B (0.1\% TFA / $99.9 \%$ acetonitrile). Polypeptide purity was confirmed by RP-HPLC with an analytical column (C18) and identified by matrix-assisted laser desorption/ionization (MALDI) (Applied Biosystem, U.S.A.) mass spectroscopy.

\section{Fluorescence-lifetime imaging microscopy.}


Based on the self-quenching of the neighboring fluorophores labelled on the GA DPRs peptide photoreleased from ADP-2, fluorescence lifetime imaging microscopy (FLIM) analysis was carried out with nanometer resolution Z-piezo objective. The laser for excitation was $488 \mathrm{~nm}$ modulated laser line with $20 \mathrm{MHz}$ repetition rate. The fluorescence lifetime of Alexa Fluor 488-labelled GADPRs peptides with different incubation time were monitored and compared. The excitation wavelength was connected by optical fiber and a band-pass filter to improve wavelength selection. Fluorescence emission from the sample went through a band-pass filter (FF05-500/25-25 for Alexa 488, Semrock) before being sent to the confocal unit with single photon counting module avalanche photodiodes APDs detectors. A water objective (PlanApo 60x, N.A. 1.2, Nikon) was used for the imaging. To avoid any perturbation to the sample, optical sectioning was achieved by the $X Y$ galvo mirror scanning and the $Z$ sectioning by moving Piezo encoded motorized device. For the cellular FLIM images, SH-SY5Y cells were cultured on a sterilized $35 \mathrm{~mm}$ glass bottom $\mu$-Dish (Ibidi). After ADP-2 treatment and photoinitiation (see below), cells were further incubated for $2 \mathrm{hr}, 12 \mathrm{hr}$, and 24 hours followed by $4 \%$ paraformaldehyde fixation. FLIM analysis was carried out essentially as described above.

\section{SH-SY5Y cells lysates A11 staining}

$1.6 \times 10^{6}$ of SH-SY $5 Y$ cells were treated with ADP-1 or ADP-3 $(3 \mu \mathrm{M})$ for 24 hours and then exposed to UV light (mercury lamp with 345-385 nm bandpass filter; average power: $8.24 \mathrm{~mW} / \mathrm{cm}^{2}$; duration: $1 \mathrm{~min}$ ). After washing with culture medium (DMEM/F12 supplemented with $10 \% \mathrm{FBS}$ ), cells were further incubated and collected the cell lysates in RIPA buffer at different time points. Cells lysates was first sonicated by UP200S (Hielscher Ultrasonics, Germany) and the total protein concentration in the resultant lysates were measured by detergent compatible (Dc) protein assay (Bio-Rad, U.S.A.). $50 \mu \mathrm{g}$ of proteins were loaded onto nitrocellulose membrane $(0.1 \mu \mathrm{m}$, GE healthcare, U.S.A.) adopted with the PR648 Slot Blot Blotting Manifold . Collected membranes were blocked by $2 \%$ bovine serum albumin, and then stained with A11 antibody (AHB0052, ThermoFisher) and GAPDH antibody (GTX627408, GeneTex), respectively. To quantitate the $A 11$ kinetics, the collected $A 11$ signals were first normalized to internal control GAPDH and the resultant signals were then compared to the signal at 0.5 -hour-incubation to learn the relative fold value.

\section{Ex vivo antibody penentrance assay}

To prepare the GA DPRs oligomers-rich samples, ADP-1 (100 $\mu \mathrm{M}$ in PBS) solution was irradiated by UV light ( $365 \mathrm{~nm}, 1200 \mu \mathrm{w} / \mathrm{cm}^{2}$, duration: $\left.1 \mathrm{~min}\right)$ and incubated for 2 hours at $37^{\circ} \mathrm{C}$. For the GA DPRs fibrilsrich samples, ADP-1 (100 $\mu \mathrm{M}$ in PBS) solution was irradiated by UV light and incubated for 24 hours at 37 ${ }^{\circ} \mathrm{C}$. For plasma membrane permeablization, $2 \times 10^{6}$ of SH-SY $5 Y$ cells were pre-treated with icy digtonin $(0.01 \%$ in PBS) for 2 minutes. The resulting nuclei were washed by PBS for three times and then treated with GA DPRs oligomers or fibrils for 2 hours. After PBS rinses, the premeabilized remains were fixed and stained with lamin B1 antibody. Confocal images of the resulting samples were captured with LSM 780 . The antibody penetrance level in the nuclei after digitonin treatment was evaluated by the number of nuclei with positive staining of Lamin B1 over total nuclei number. 


\section{Calcein-leakage assay.}

For preparation of calcein-containing liposome, 1,2-Ditetradecanoyl-sn-glycero-3-phospho-(1'-rac-glycerol) (sodium salt) (DMPG) (3.3 mg), 1,2-Dimyristoyl-snglycero-3-phosphocholine (DMPC) (5.0 mg), and Cholesterol $(2.5 \mathrm{mg})$ were dissolved in 1:1 chloroform/methanol. Nitrogen gas and lyophilizer was used to remove the solvents from the sample. The dried lipid was rehydrated by $1 \mathrm{ml}$ of $50 \mathrm{mM}$ calceincontaining PBS (phosphate buffered saline) titrated with potassium hydroxide to $\mathrm{pH}$ 7.0. The solution was sonicated for 1 hour. Freeze-thaw cycles in liquid nitrogen and a $70^{\circ} \mathrm{C}$ hot plate were processed for 7 times. The suspension was passed back and forth continuously through an Avanti Mini-Extruder with two stacked $100 \mathrm{~nm}$ polycarbonate membranes (Avanti Polar Lipid, Inc). Unencapsulated calcein was removed by self-packed size-exclusion column with Sepharose CL-4B gel filtration medium (Sigma). For measuring the fluorescence intensity, peptides $(100 \mu \mathrm{M})$ were freshly prepared and then added into the liposome solution to a final concentration of $0.7 \mathrm{mM}$ lipid and $50 \mu \mathrm{M}$ peptide. For collecitng GA DPRs fibrils, the irradiated ADP-1 was incubated for 1 day , centrifuged. The pellet was re-suspended before adding to liposome solution. The liposome and peptide mixtures were incubated at $37^{\circ} \mathrm{C}$ with $1400 \mathrm{rpm}$ shaking in an Eppendorf Thermomixer® mixer/ incubator. Fluorescence was measured at excitation/emission $490 / 520 \mathrm{~nm}$. The percentage of fluorescence intensity is defined as $(\mathrm{FP}-\mathrm{FL}) /(\mathrm{FT}-\mathrm{FL})$, where $\mathrm{FP}$ is the fluorescence signal after the addition of peptides, $\mathrm{FL}$ is the fluorescence for liposome only, and FT is the fluorescence signal obtained after addition of $5 \%$ of Triton $\mathrm{X}-100$.

\section{Declarations}

\section{Author Contributions}

J.J.H. and H.M.C. designed the study. H.M.C. designed and prepared the probes and peptides. H.M.C. performed biophysical and biochemical experiments. H.M.C. carried out TEM experiments. I.J.H., K.T.H., R.Y.H., J.C.H., D.A., C.C.L., Y.A.H., and H.M.C. carried out and guided cellular experiments. K.T.H., P.C.L., and R.Y.H. carried out imaging experiments. Y.A.H. and E.H. carried out cortical neuron experiments. H.M.C., R.Y.H., Y.A.H., E.H., C.C.L, and J.J.H. analyzed the data. H.M.C. and E.H. wrote the manuscript. J.J.H. and C.C.L. advised the manuscript.

\section{Conflicts of Interest}

The authors declare no conflict in interest.

\section{Acknowledgment}

We thank Academia Sinica (AS-TP-106-L13, AS-CDA-109-M09) and the Ministry of Science and Technology (MOST 106-2628-M-001-002-MY3 and MOST 109-2113-M-001-029), Taiwan, for financial support. We thank Dr. Wen Zhi-Hong (Department of Marine Biotechnology and Resource, National Sun Yat-sen University) for generously providing the SH-SY5Y cells. We also appreciate Dr. Ya-Jen Cheng 
(Neuroscience Program, Academia Sinica), Sue-Ping Lee and Shu-Mei Huang (Imaging Core, Institute of Molecular Biology, Academia Sinica), and Dr. Po-Yen Lin and Yao-Kwan Huang (Biological electron microscopy core facility, Institute of Cellular and Organismic Biology, Academia Sinica) for their technical support. The core facility is funded by Academia Sinica Core Facility and Innovative Instruments Project (AS-CFII-108-119). We thank Academia Sinica Neuroscience Core Facility for confocal imaging analysis and technical support. The core facility is funded by Academia Sinica Core Facility and Innovative Instrument Project (AS-CFII-108-106). We thank Dr. Yijuang Chern (Institute of Biomedical Science, Academia Sinica) and Dr. Yu-Ju Chen (Institute of Chemistry, Academia Sinica) for the constructive comments and suggestions. This manuscript was edited by Wallace Academic Editing.

\section{Footnotes}

The supplementary information is available online Supplementary figures and Methods details (PDF)

\section{References}

1 DeJesus-Hernandez, M. et al. Expanded GGGGCC hexanucleotide repeat in noncoding region of C9ORF72 causes chromosome 9p-linked FTD and ALS. Neuron 72, 245-256, doi:10.1016/j.neuron.2011.09.011 (2011).

2 Renton, Alan E. et al. A Hexanucleotide Repeat Expansion in C9ORF72 Is the Cause of Chromosome 9p21-Linked ALS-FTD. Neuron 72, 257-268, doi:https://doi.org/10.1016/j.neuron.2011.09.010 (2011).

3 Gijselinck, I. et al. A C9orf72 promoter repeat expansion in a Flanders-Belgian cohort with disorders of the frontotemporal lobar degeneration-amyotrophic lateral sclerosis spectrum: a gene identification study. The Lancet Neurology 11, 54-65, doi:https://doi.org/10.1016/S1474-4422(11)70261-7 (2012).

4 Haeusler, A. R., Donnelly, C. J. \& Rothstein, J. D. The expanding biology of the C9orf72 nucleotide repeat expansion in neurodegenerative disease. Nat. Rev. Neurosci. 17, 383, doi:10.1038/nrn.2016.38 https://www.nature.com/articles/nrn.2016.38\#supplementary-information (2016).

5 Brown, R. H. \& Al-Chalabi, A. Amyotrophic Lateral Sclerosis. New Engl. J. Med. 377, 162-172, doi:10.1056/NEJMra1603471 (2017).

6 Balendra, R. \& Isaacs, A. M. C9orf72-mediated ALS and FTD: multiple pathways to disease. Nat. Rev. Neurol. 14, 544-558, doi:10.1038/s41582-018-0047-2 (2018).

7 Donnelly, Christopher J. et al. RNA Toxicity from the ALS/FTD C9ORF72 Expansion Is Mitigated by Antisense Intervention. Neuron 80, 415-428, doi:https://doi.org/10.1016/j.neuron.2013.10.015 (2013).

8 Lagier-Tourenne, C. et al. Targeted degradation of sense and antisense C9orf72 RNA foci as therapy for ALS and frontotemporal degeneration. Proc. Natl. Acad. Sci. U. S. A. 110, E4530, 
doi:10.1073/pnas.1318835110 (2013).

9 Sareen, D. et al. Targeting RNA Foci in iPSC-Derived Motor Neurons from ALS Patients with a C9ORF72 Repeat Expansion. Sci. Transl. Med. 5, 208ra149, doi:10.1126/scitransImed.3007529 (2013).

10 Reddy, K., Zamiri, B., Stanley, S. Y. R., Macgregor, R. B. \& Pearson, C. E. The Disease-associated r(GGGGCC)n Repeat from the C9orf72 Gene Forms Tract Length-dependent Uni- and Multimolecular RNA G-quadruplex Structures. J. Biol. Chem. 288, 9860-9866, doi:10.1074/jbc.C113.452532 (2013).

11 Conlon, E. G. et al. The C9ORF72 GGGGCC expansion forms RNA G-quadruplex inclusions and sequesters hnRNP H to disrupt splicing in ALS brains. eL ife 5, e17820, doi:10.7554/eLife.17820 (2016).

12 Yamakawa, M. et al. Characterization of the dipeptide repeat protein in the molecular pathogenesis of c9FTD/ALS. Hum. Mol. Genet. 24, 1630-1645, doi:10.1093/hmg/ddu576 (2015).

13 Lee, K. H. et al. C9orf72 Dipeptide Repeats Impair the Assembly, Dynamics, and Function of Membrane-Less Organelles. Cell 167, 774-788 e717, doi:10.1016/j.cell.2016.10.002 (2016).

14 Zhang, Y.-J. et al. C9ORF72 poly(GA) aggregates sequester and impair HR23 and nucleocytoplasmic transport proteins. Nat. Neurosci. 19, 668, doi:10.1038/nn.4272

https://www.nature.com/articles/nn.4272\#supplementary-information (2016).

15 Shi, K. Y. et al. Toxic PRn poly-dipeptides encoded by the C9orf72 repeat expansion block nuclear import and export. Proc. Natl. Acad. Sci. U. S. A. 114, E1111-E1117, doi:10.1073/pnas.1620293114 (2017).

16 Yin, S. et al. Evidence that C9ORF72 Dipeptide Repeat Proteins Associate with U2 snRNP to Cause Mis-splicing in ALS/FTD Patients. Cell Rep 19, 2244-2256, doi:10.1016/j.celrep.2017.05.056 (2017).

17 Freibaum, B. D. \& Taylor, J. P. The Role of Dipeptide Repeats in C90RF72-Related ALS-FTD. Front. Mol. Neurosci. 10, doi:10.3389/fnmol.2017.00035 (2017).

18 Mori, K. et al. The C9orf72 GGGGCC repeat is translated into aggregating dipeptide-repeat proteins in FTLD/ALS. Science 339, 1335-1338, doi:10.1126/science.1232927 (2013).

19 Ash, Peter E. A. et al. Unconventional Translation of C90RF72 GGGGCC Expansion Generates Insoluble Polypeptides Specific to c9FTD/ALS. Neuron 77, 639-646, doi:https://doi.org/10.1016/j.neuron.2013.02.004 (2013).

20 Troakes, C. et al. An MND/ALS phenotype associated with C9orf72 repeat expansion: Abundant p62-positive, TDP-43-negative inclusions in cerebral cortex, hippocampus and cerebellum but without associated cognitive decline. Neuropathology 32, 505-514, doi:10.1111/j.1440-1789.2011.01286.x (2012). 
21 Lee, Y.-B. et al. C9orf72 poly GA RAN-translated protein plays a key role in amyotrophic lateral sclerosis via aggregation and toxicity. Hum. Mol. Genet. 26, 4765-4777, doi:10.1093/hmg/ddx350 (2017).

22 Mann, D. M. A. et al. Dipeptide repeat proteins are present in the $\mathrm{p} 62$ positive inclusions in patients with frontotemporal lobar degeneration and motor neurone disease associated with expansions in C90RF72. Acta Neuropathol Commun 1, 68, doi:10.1186/2051-5960-1-68 (2013).

23 Davidson, Y. S. et al. Brain distribution of dipeptide repeat proteins in frontotemporal lobar degeneration and motor neurone disease associated with expansions in C9ORF72. Acta Neuropathol Commun 2, 70, doi:10.1186/2051-5960-2-70 (2014).

24 Edbauer, D. \& Haass, C. An amyloid-like cascade hypothesis for C9orf72 ALS/FTD. Current Opinion in Neurobiology 36, 99-106, doi:https://doi.org/10.1016/j.conb.2015.10.009 (2016).

25 Chang, Y.-J., Jeng, U.-S., Chiang, Y.-L., Hwang, I.-S. \& Chen, Y.-R. The Glycine-Alanine Dipeptide Repeat from C9orf72 Hexanucleotide Expansions Forms Toxic Amyloids Possessing Cell-to-Cell Transmission Properties. J. Biol. Chem. 291, 4903-4911, doi:10.1074/jbc.M115.694273 (2016).

26 Zhang, Y.-J. et al. Aggregation-prone c9FTD/ALS poly(GA) RAN-translated proteins cause neurotoxicity by inducing ER stress. Acta Neuropatho/ 128, 505-524, doi:10.1007/s00401-014-1336-5 (2014).

27 May, S. et al. C9orf72 FTLD/ALS-associated Gly-Ala dipeptide repeat proteins cause neuronal toxicity and Unc119 sequestration. Acta Neuropatho/ 128, 485-503, doi:10.1007/s00401-014-1329-4 (2014).

28 Cline, E. N., Bicca, M. A., Viola, K. L. \& Klein, W. L. The Amyloid- $\beta$ Oligomer Hypothesis: Beginning of the Third Decade. J Alzheimers Dis 64, S567-S610, doi:10.3233/JAD-179941 (2018).

29 Yamakawa, M. et al. Characterization of the dipeptide repeat protein in the molecular pathogenesis of c9FTD/ALS. Hum Mol Genet 24, 1630-1645, doi:10.1093/hmg/ddu576 (2015).

30 Nonaka, T. et al. C9ORF72 dipeptide repeat poly-GA inclusions promote intracellular aggregation of phosphorylated TDP-43. Hum. Mol. Genet. 27, 2658-2670, doi:10.1093/hmg/ddy174 (2018).

31 Morgado, I. et al. Molecular basis of $\beta$-amyloid oligomer recognition with a conformational antibody fragment. Proceedings of the National Academy of Sciences 109, 12503, doi:10.1073/pnas.1206433109 (2012).

32 Kayed, R. et al. Fibril specific, conformation dependent antibodies recognize a generic epitope common to amyloid fibrils and fibrillar oligomers that is absent in prefibrillar oligomers. Mol. Neurodegener. 2, 18, doi:10.1186/1750-1326-2-18 (2007). 
33 Chen, W. et al. Fluorescence Self-Quenching from Reporter Dyes Informs on the Structural Properties of Amyloid Clusters Formed in Vitro and in Cells. Nano Lett. 17, 143-149, doi:10.1021/acs.nanolett.6b03686 (2017).

34 Chou, C.-C. et al. TDP-43 pathology disrupts nuclear pore complexes and nucleocytoplasmic transport in ALS/FTD. Nat. Neurosci. 21, 228-239, doi:10.1038/s41593-017-0047-3 (2018).

35 Lin, Y. et al. Toxic PR Poly-Dipeptides Encoded by the C9orf72 Repeat Expansion Target LC Domain Polymers. Cell 167, 789-802 e712, doi:10.1016/j.cell.2016.10.003 (2016).

36 Cavazza, T. \& Vernos, I. The RanGTP Pathway: From Nucleo-Cytoplasmic Transport to Spindle Assembly and Beyond. Frontiers in Cell and Developmental Biology 3, doi:10.3389/fcell.2015.00082 (2016).

37 Lowe, A. R. et al. Importin- $\beta$ modulates the permeability of the nuclear pore complex in a Randependent manner. elife 4, e04052, doi:10.7554/eLife.04052 (2015).

38 Paonessa, F. et al. Microtubules Deform the Nuclear Membrane and Disrupt Nucleocytoplasmic Transport in Tau-Mediated Frontotemporal Dementia. Cell Reports 26, 582-593.e585, doi:https://doi.org/10.1016/j.celrep.2018.12.085 (2019).

39 Liu, K.-Y. et al. Disruption of the nuclear membrane by perinuclear inclusions of mutant huntingtin causes cell-cycle re-entry and striatal cell death in mouse and cell models of Huntington's disease. Hum. Mol. Genet. 24, 1602-1616, doi:10.1093/hmg/ddu574 (2015).

40 Diez, L. \& Wegmann, S. Nuclear Transport Deficits in Tau-Related Neurodegenerative Diseases. Front. Neurol. 11, 1056-1056, doi:10.3389/fneur.2020.01056 (2020).

41 Worman, H. J. \& Courvalin, J. C. in Int. Rev. Cytol. Vol. 246 231-279 (Academic Press, 2005).

42 Fusco, G. et al. Structural basis of membrane disruption and cellular toxicity by a-synuclein oligomers. Science 358, 1440, doi:10.1126/science.aan6160 (2017).

43 Fan, H. Y. \& Heerklotz, H. Digitonin does not flip across cholesterol-poor membranes. Journal of Colloid and Interface Science 504, 283-293, doi:https://doi.org/10.1016/j.jcis.2017.05.034 (2017).

44 Liu, G. C.-H. et al. Delineating the membrane-disrupting and seeding properties of the TDP-43 amyloidogenic core. Chem. Commun. 49, 11212-11214, doi:10.1039/C3CC46762G (2013).

45 Neumann, M. et al. Ubiquitinated TDP-43 in Frontotemporal Lobar Degeneration and Amyotrophic Lateral Sclerosis. Science 314, 130, doi:10.1126/science.1134108 (2006).

46 Gao, J., Wang, L., Huntley, M. L., Perry, G. \& Wang, X. Pathomechanisms of TDP-43 in neurodegeneration. J. Neurochem. 146, 7-20, doi:10.1111/jnc.14327 (2018). 
47 Hartmann, H. et al. Cytoplasmic poly-GA aggregates impair nuclear import of TDP-43 in C9orf72 ALS/FTLD. Hum. Mol. Genet. 26, 790-800, doi:10.1093/hmg/ddw432 (2016).

48 Mackenzie, I. R. et al. Dipeptide repeat protein pathology in C9ORF72 mutation cases: clinicopathological correlations. Acta Neuropatho/ 126, 859-879, doi:10.1007/s00401-013-1181-y (2013).

49 Ayala, Y. M. et al. Structural determinants of the cellular localization and shuttling of TDP-43. J. Cell Sci. 121, 3778, doi:10.1242/jcs.038950 (2008).

50 Davidson, Y. et al. Neurodegeneration in frontotemporal lobar degeneration and motor neurone disease associated with expansions in C9orf72 is linked to TDP-43 pathology and not associated with aggregated forms of dipeptide repeat proteins. Neuropathol. Appl. Neurobiol. 42, 242-254, doi:10.1111/nan.12292 (2016).

51 Mackenzie, I. R. A. et al. Quantitative analysis and clinico-pathological correlations of different dipeptide repeat protein pathologies in C9ORF72 mutation carriers. Acta Neuropatho/ 130, 845-861, doi:10.1007/s00401-015-1476-2 (2015).

52 Chew, J. et al. C90RF72 repeat expansions in mice cause TDP-43 pathology, neuronal loss, and behavioral deficits. Science 348, 1151, doi:10.1126/science.aaa9344 (2015).

53 Ichikawa, M., Muramoto, K., Kobayashi, K., Kawahara, M. \& Kuroda, Y. Formation and maturation of synapses in primary cultures of rat cerebral cortical cells: an electron microscopic study. Neurosci. Res. 16, 95-103, doi:https://doi.org/10.1016/0168-0102(93)90076-3 (1993).

\section{Figures}




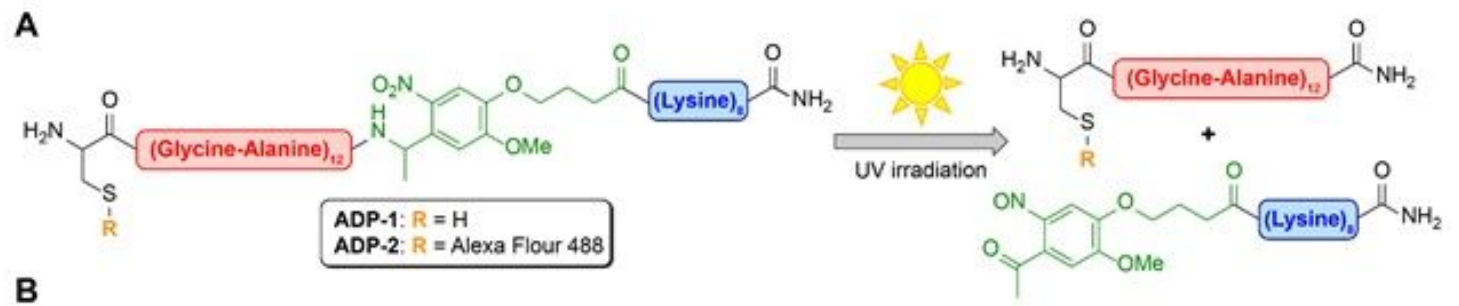

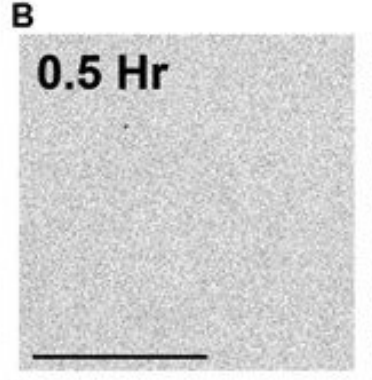

C

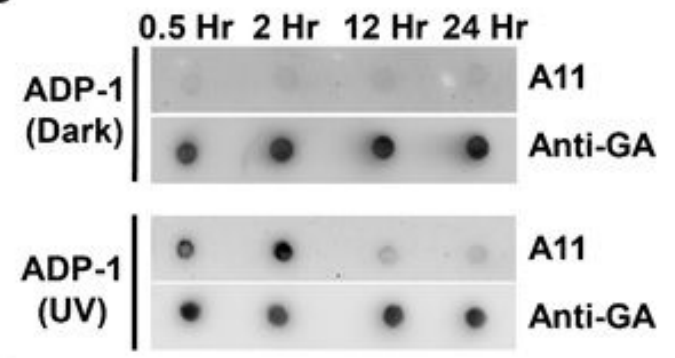

$\mathbf{E}$
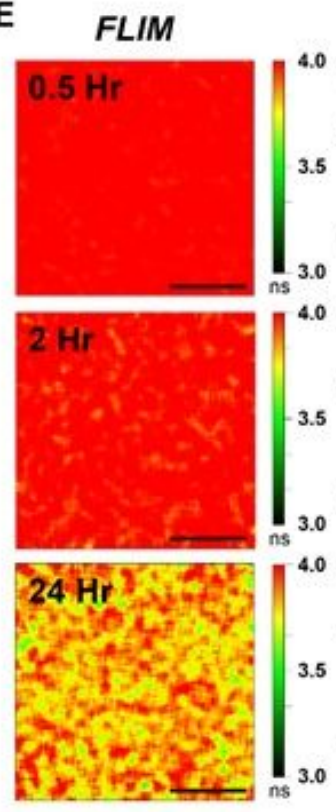

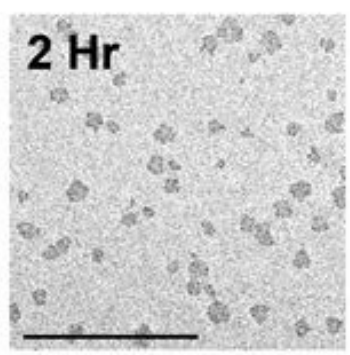

A11

Anti-GA

Histogram
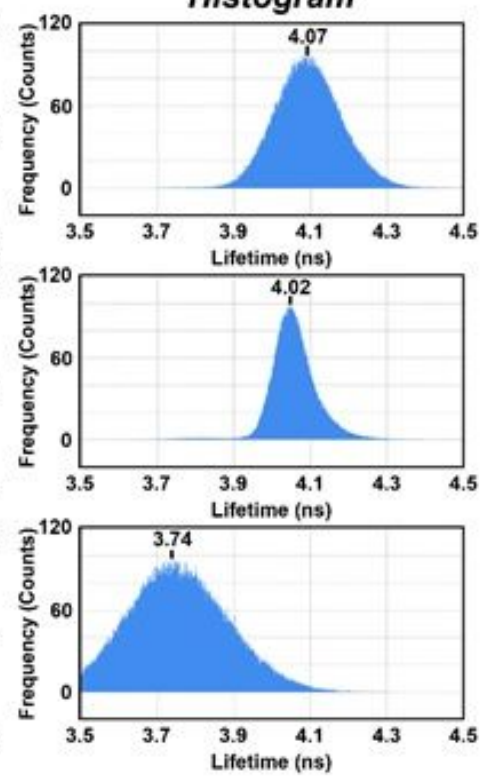
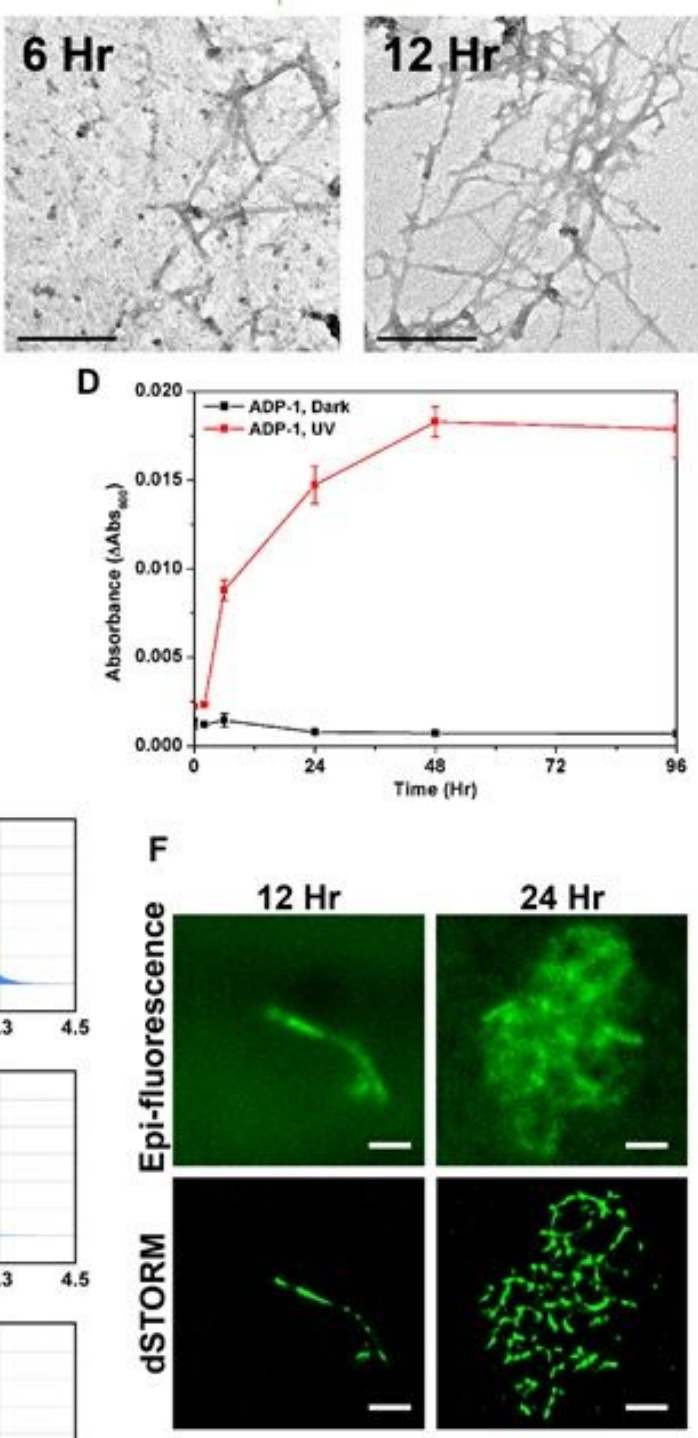

$24 \mathrm{Hr}$

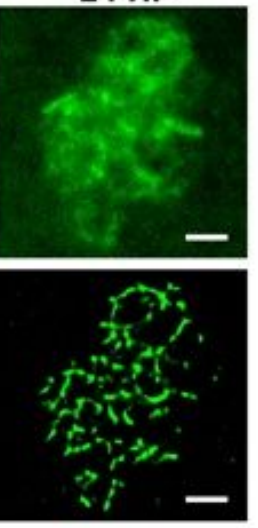

\section{Figure 1}

Diagram of ADP probes and its biophysical characterization. (A) Structure of ADP-1 and ADP-2 probes and the scheme of their photo-liberatiing reaction. (B) Time-course transmission electron microscope analysis of ADP-1 $(50 \mu \mathrm{M})$. Scale bars indicate $200 \mathrm{~nm}$. (C) Dot blot analysis of ADP-1-induced GA DPRs over time. The Sample were incubated until the indicated time and applied on membrane. Membranes were stained with anti-GA DPRs antibody and A11 antibody. N=3. (D) Time-course turbidity 
measurements of irradiated (wavelength: $365 \mathrm{~nm}$, power density: $32 \mathrm{~mW} / \mathrm{cm} 2$, duration: 1 minute) (red) and unirradiated (black) ADP-1 $(50 \mu \mathrm{M})$. Data are collected at 0.5th, 2nd, 6th, 12th, 24th, 48th, and 96th hour (S.D., $n=3)(E)$ Fluorescence lifetime images and the histogram of the photoinitiated ADP-2 (5 $\mu M)$. Images were taken at the 0.5 th, 2 nd, and 24 th hour of incubation; scale bars indicate $5 \mu \mathrm{m}$. (F) Epifluorescence and direct stochastic optical reconstruction microscopy (dSTORM) images of the photoinitiated ADP-2 $(50 \mu \mathrm{M})$ after. Images were acquired at the 12th and 24th hours of incubation; scale bars indicate $10 \mu \mathrm{m}$.

A
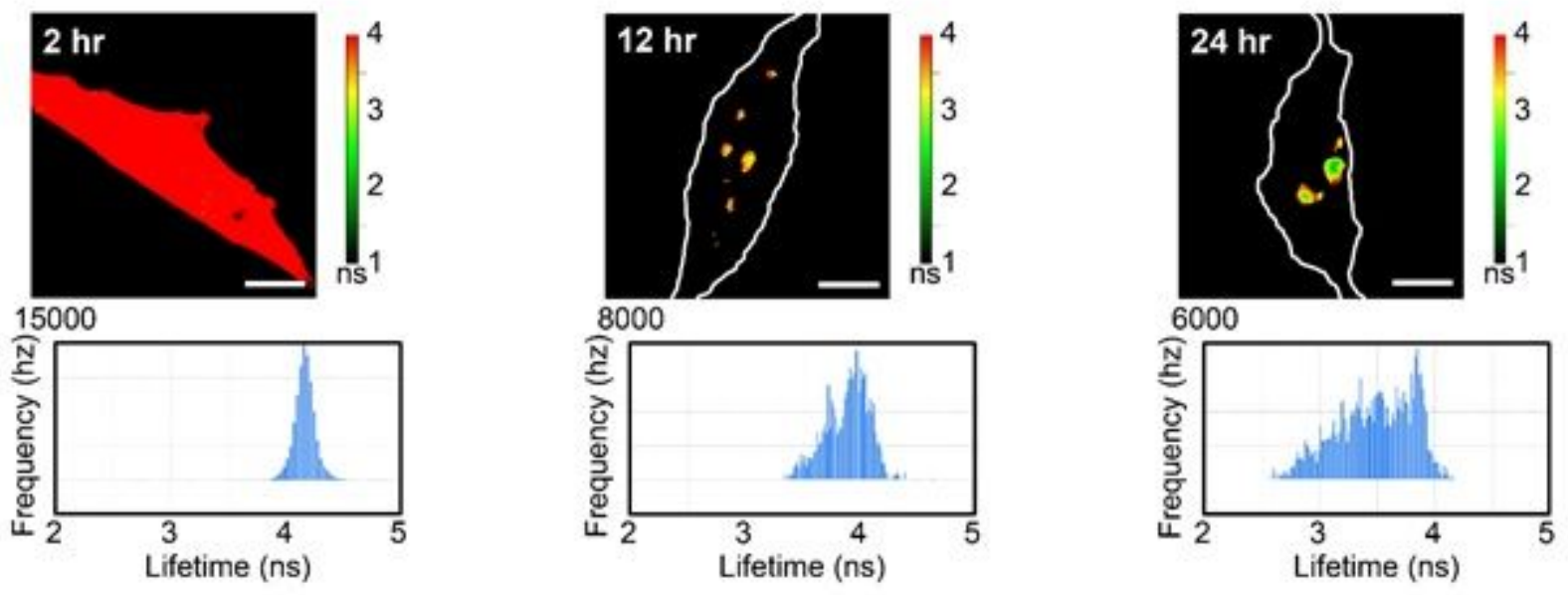

B

$0.5 \mathrm{Hr} 2 \mathrm{Hr} \quad 6 \mathrm{Hr} 12 \mathrm{Hr} 24 \mathrm{Hr}$
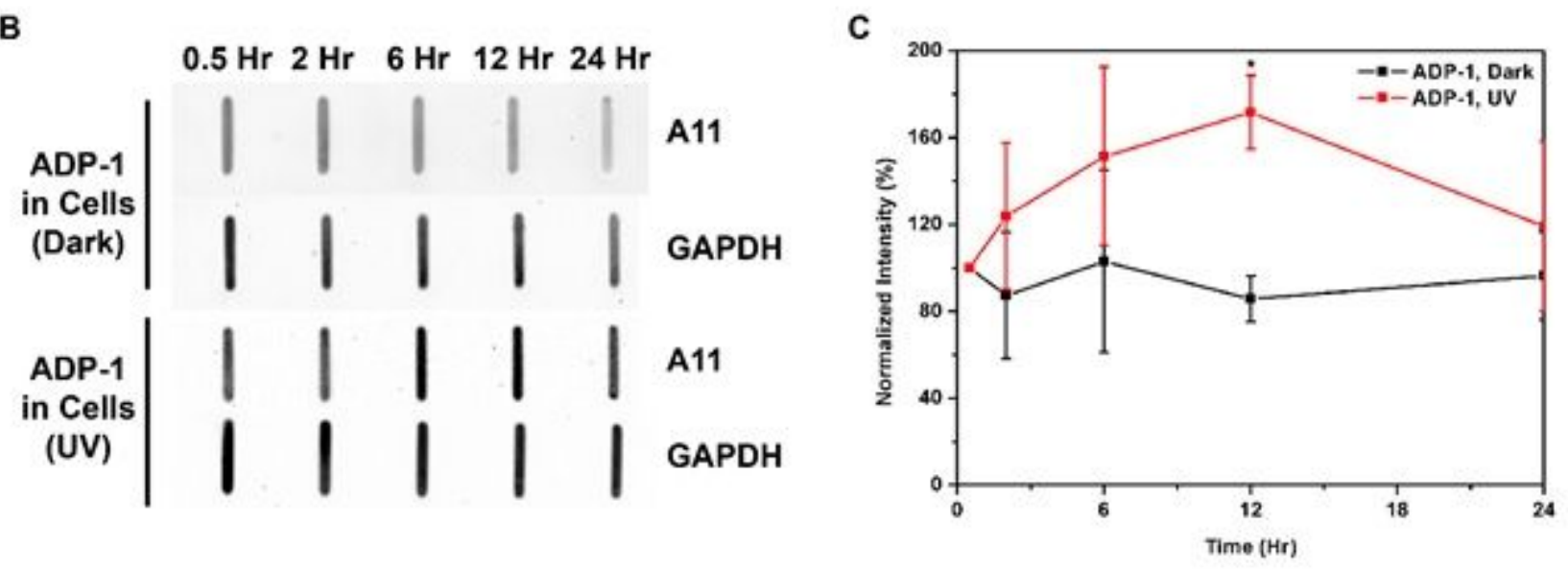

Figure 2

GA DPRs delivered by ADP-1 and 2 probes induce nuclear transport defects. (A) Time-course fluorescence-lifetime images and histograms of ADP-2 in SH-SY5Y cells. $1 \mu \mathrm{M}$ ADP-2 were treated to cells and photoinitiated by UV light (wavelength: $335-379 \mathrm{~nm}$, power density: $\leq 8.24 \mathrm{~mW} / \mathrm{cm} 2$, duration: 1 minute). Cell periphery was contoured with white line in 12th and 24th hour, respectively. Images were taken at 2nd, 12th, 24th hour after UV illumination; scale bars indicate $5 \mu \mathrm{m}$. (B) A11 immunoblotting with cell lysates from SH-SY5Y cells received ADP-1 $(1 \mu \mathrm{M})$ treatment with or without photoinitiation. After photoinitiation, Cell lysates at different time points were harvested and analyzed. (C) Quantification analysis of A11 immunoblot of SH-SY5Y cell lysates. The signal of A11 stainging was first normalized to 
GAPDH staining and then compared to signal at 0.5 hour incubation. Three indepedent experiement were carried out. * indicate significant, $\mathrm{p}<0.05$ (analyzed by student's t-test)
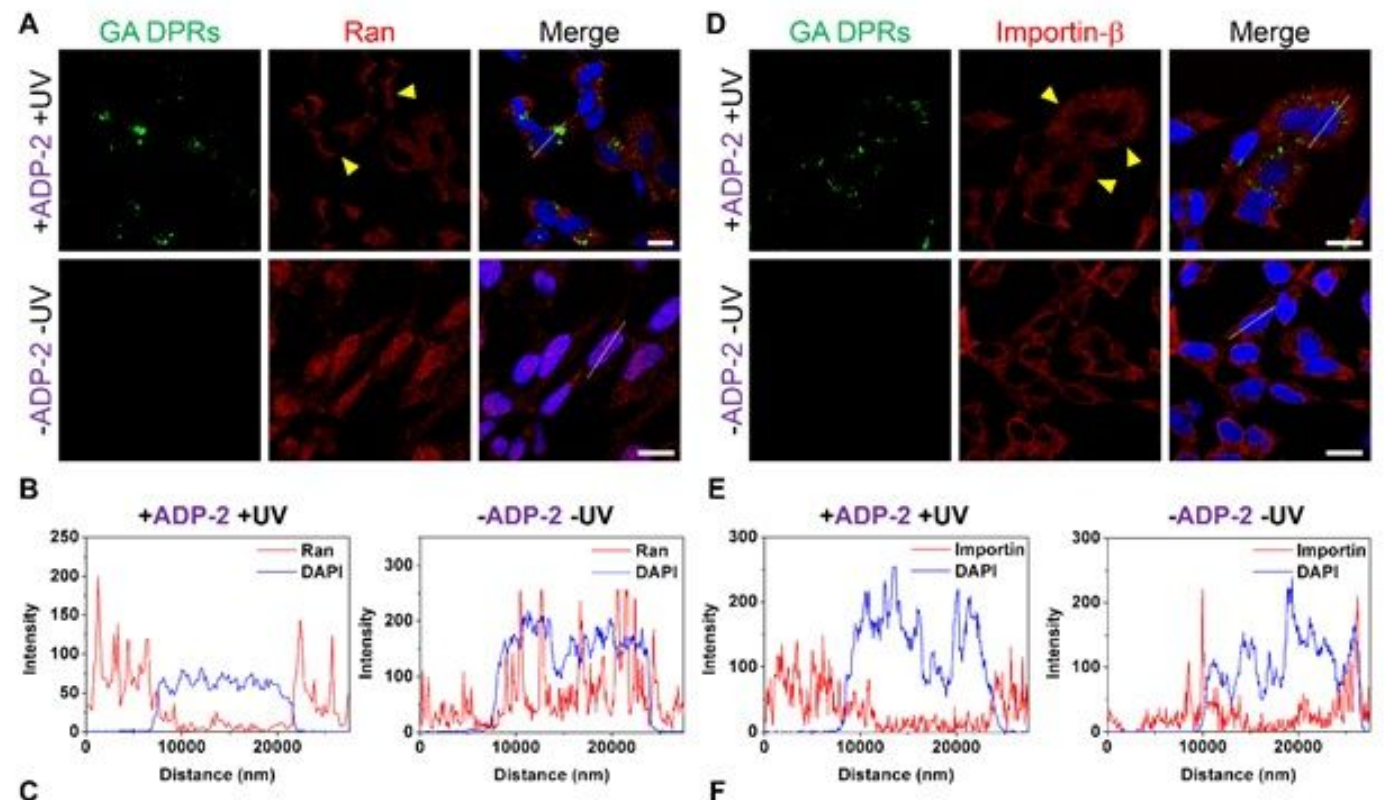

E
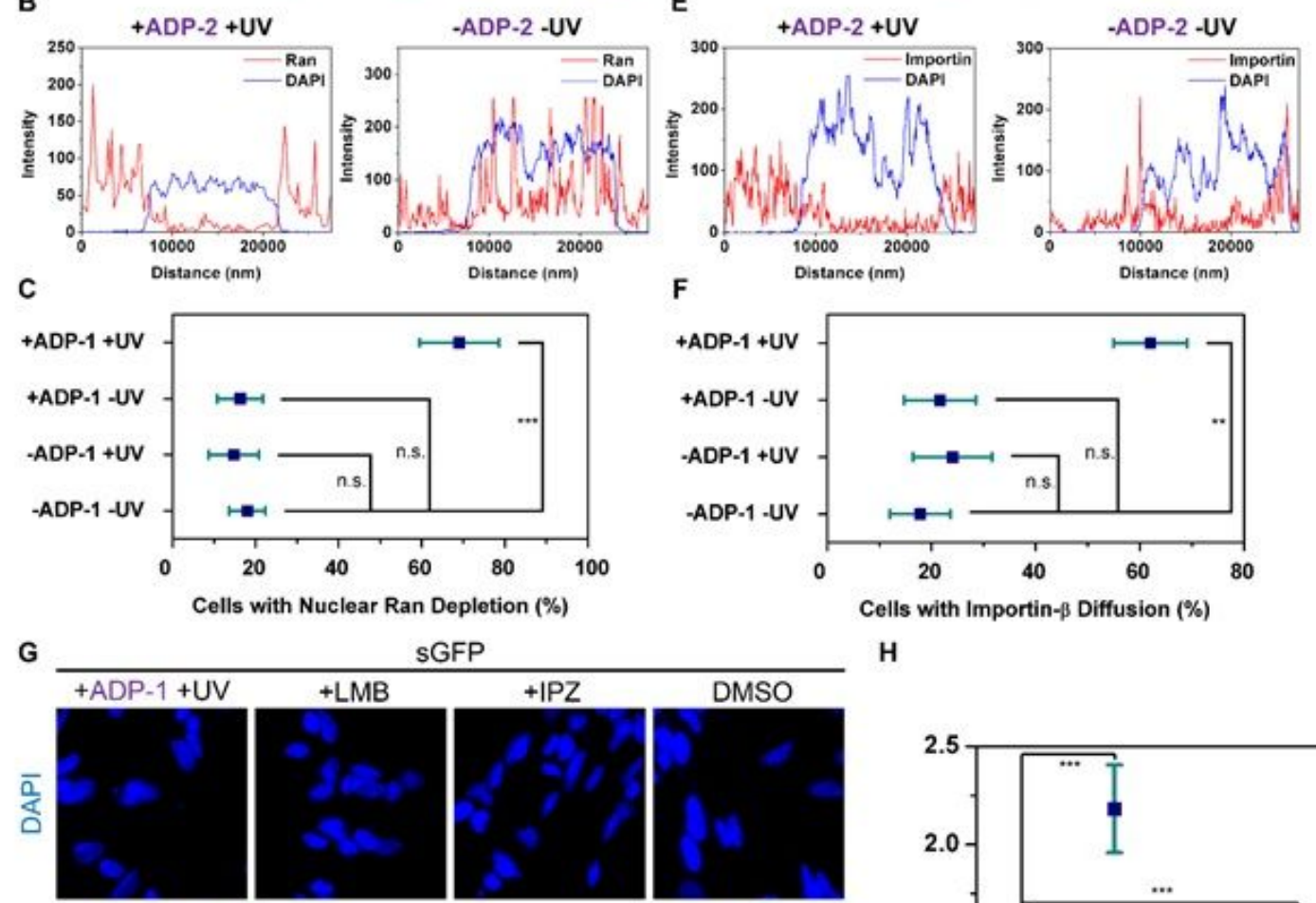

SGFP

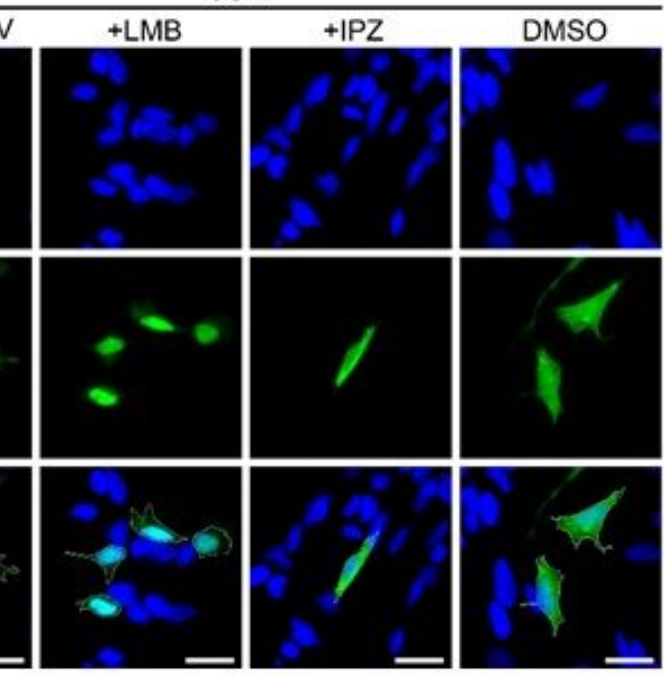

$\mathrm{H}$
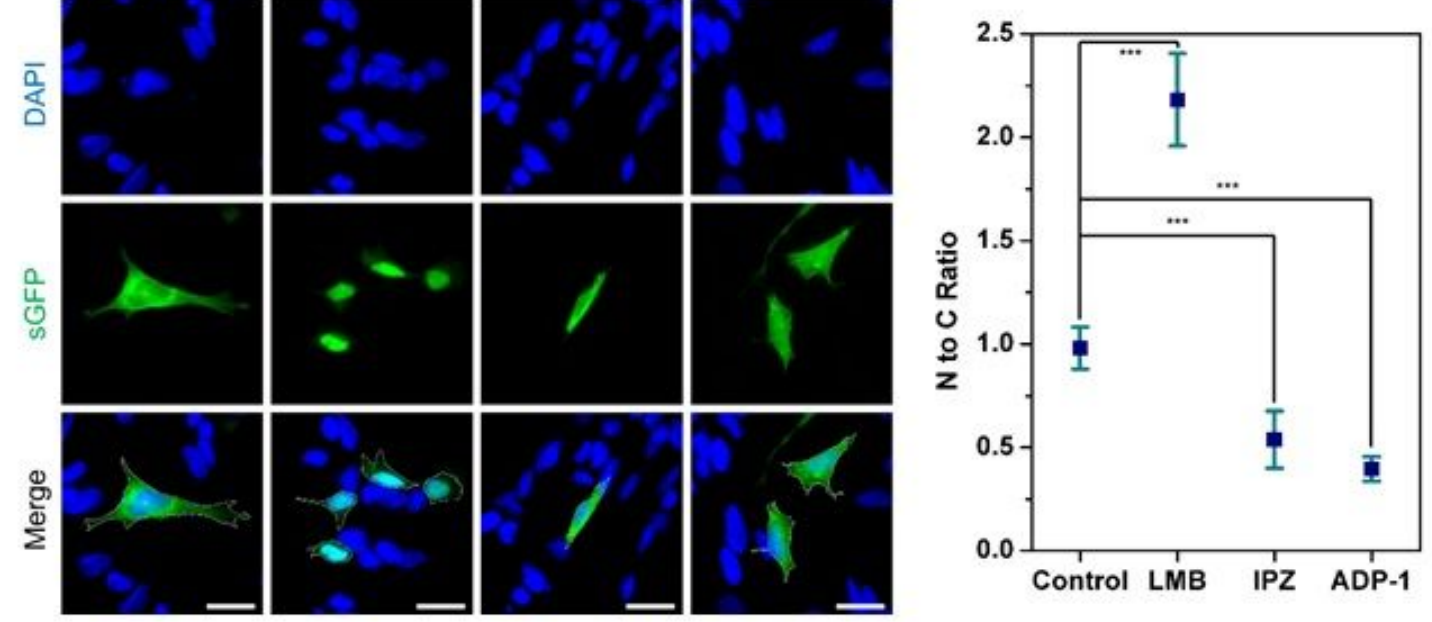

\section{Figure 3}

Photoinitiated GA DPRs impaired nucleocytoplasmic transport in human neuroblastoma cells. (A) Immunofluorescence images of Ran protein (red) in ADP-2 photoinitiated (+ADP-2 +UV) or control cells. GA DPRs aggregates (green)-rich SH-SY5Y cells. Cells were treated with ADP-2 (1 $\mu \mathrm{M})$ and then exposure 
to UV light (wavelength: $335-379 \mathrm{~nm}$, power density: $\leq 8.24 \mathrm{~mW} / \mathrm{cm} 2$, duration: 1 minute). Yellow arrows indicate the cells with nuclear Ran depletion. White dash line indicated the region for fluorescence intensity profiling. Scale bars indicate $10 \mu \mathrm{m}$. (B) Fluorescence intensity profile of selected cells in Figure 3A. Blue curve indicated DAPI channel and red curve indicated Ran channel. (C) Quantification analysis of SH-SY5Y cells with nuclear ran protein depletion. Three independent experiments were analyzed. More than 30 neurons were counted and analyzed in each groups. (D) Immunofluorescence images of importin- $\beta$ (red) in SH-SY5Y after ADP-2 $(1 \mu \mathrm{M})$ and irradiation treatment. Yellow arrows indicate the cells with importin- $\beta$ mislocalization. White dash line indicated the region for fluorescence intensity profiling. Scale bars indicate $10 \mu \mathrm{m}$. (E) Fluorescence intensity profile of selected cells in Figure 3D. Blue curve indicated DAPI channel and red curve indicated importin- $\beta$ channel. $(F)$ Quantification analysis of SHSY5Y cells with importin- $\beta$ mislocalization. Three independent experiments were analyzed. More than 35 neurons were counted and analyzed in each groups. (G) Immunofluorescence images of sGFPtransfected SH-SY5Y cells after ADP-1 treatment $(1 \mu \mathrm{M})$ and UV-irradiation. Corresponding nuclear importation (Importazole, IPZ, $40 \mu \mathrm{M}$ ) and exportation (Leptomycin B, LMB, $20 \mathrm{nM}$ ) inhibitors were used here as reference groups, respectively. Scale bars indicate $10 \mu \mathrm{m}$. (H) Quantification analysis of nuclearto-cytoplasmic ratio of SGFP reporter in SH-SY5Y. (cell counting number for Control: $n=38, L M B: n=36$, IPZ: $n=31$, ADP-1: $n=38$ ). n.s. indicate not significant; * significant, $p<0.05$; ** significant, $p<0.01$; *** significant, $p<0.001$ (analyzed by one-way ANOVA). 


\section{A}

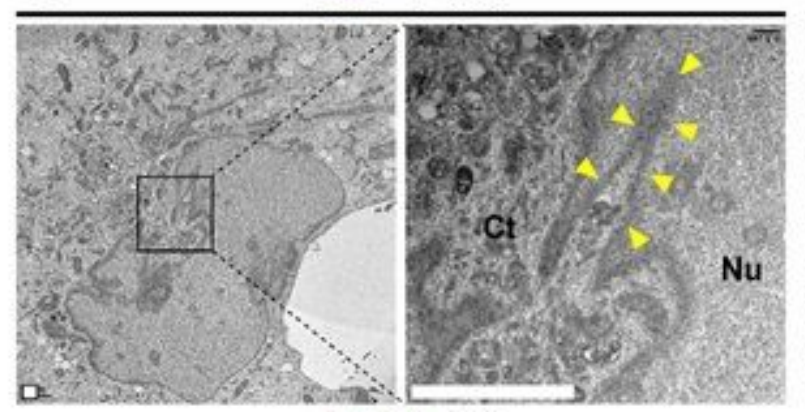

+ADP-1 -UV

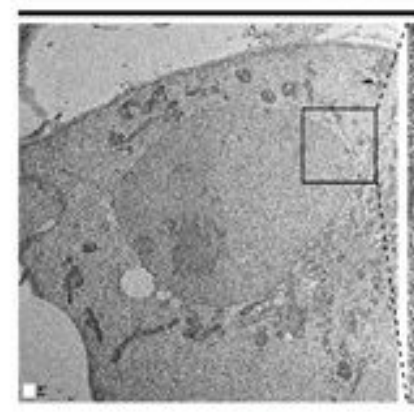

B
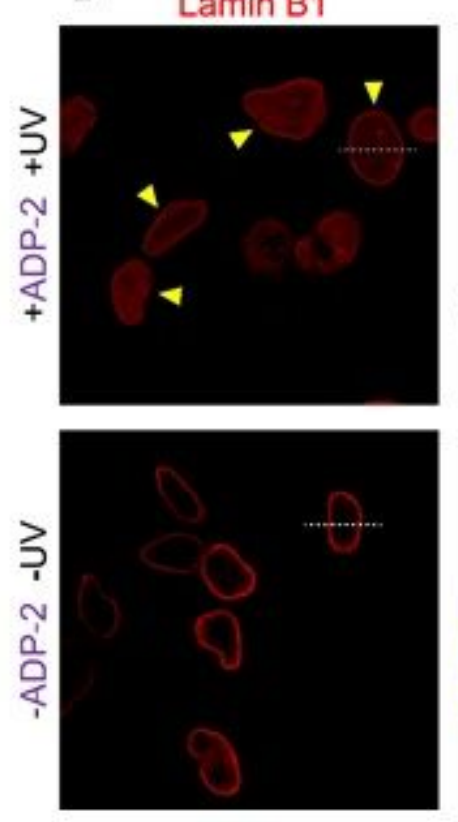

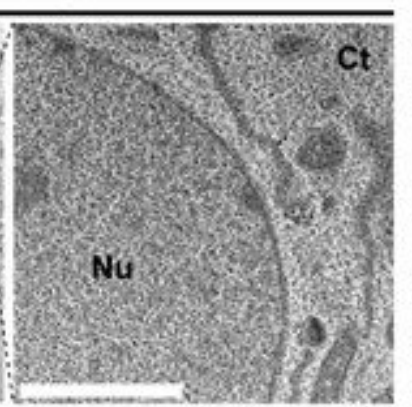

Merge
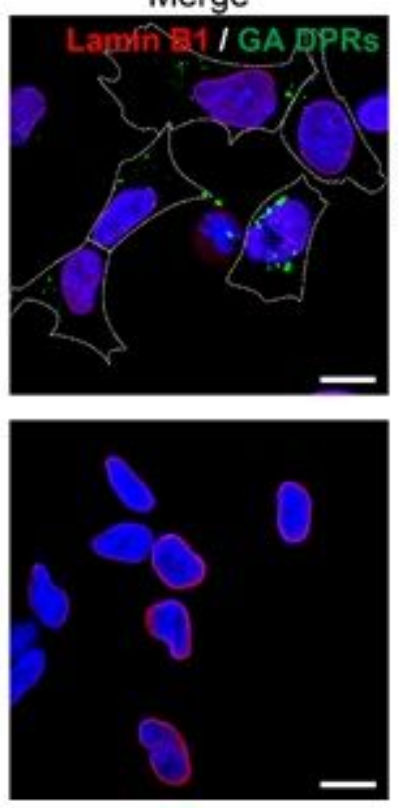

-ADP-1 -UV

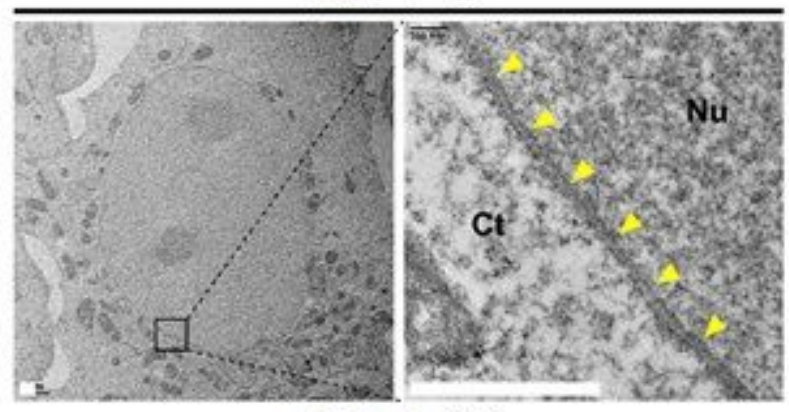

$-A D P-1+U V$

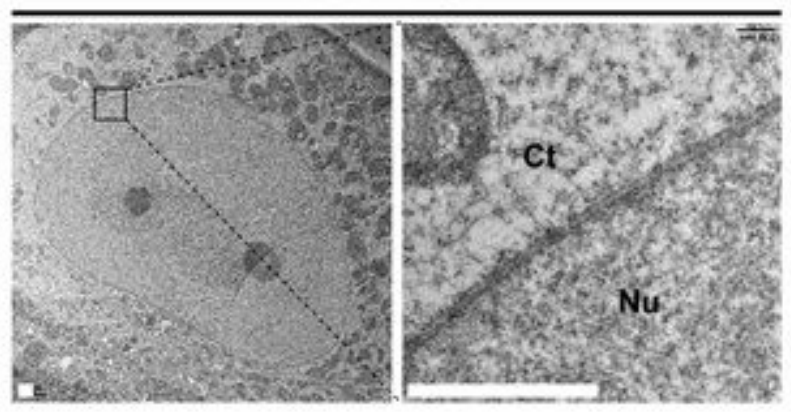

C
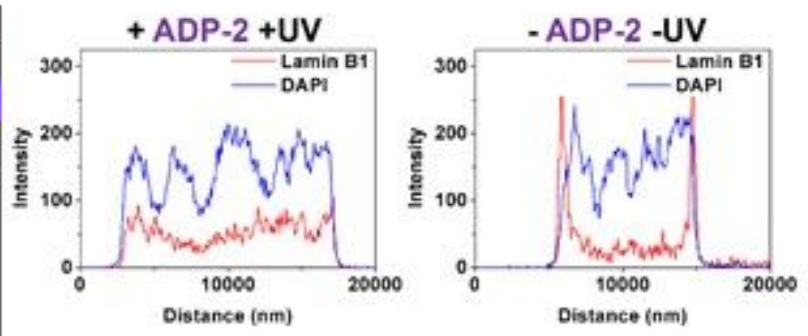

D

Cells with Abnormal Lamin B1 Distribution (\%)

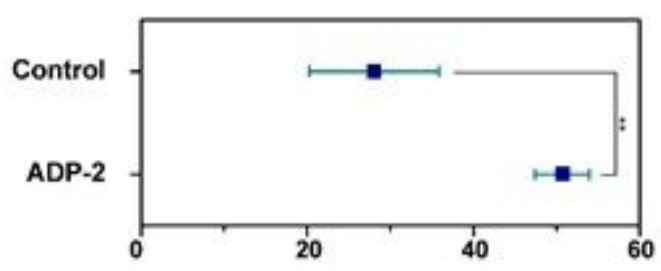

\section{Figure 4}

GA DPRs disrupts nuclear membrane and lamina. (A) Transmission electron microscope images of in Cos-7 cells with ADP-1 treatment and photoinitiation, ADP-1 treatment only, photoinitiation only, and not treatment. Yellow arrows indicate the nuclear membrane. Black squares indicate the view of zoom-in images. Scale bar indicates $1 \mu \mathrm{m}$. (B) Immunofluorescence images of lamin B1 (red) in GA DPRs (green)rich SH-SY5Y. Cells were treated with ADP-2 $(1 \mu \mathrm{M})$ and photoinitiation. Yellow arrows indicate the abnormal lamin B1 distribution. White dash line in the lamin B1 channel indicated the region for fluorescence intensity profiling. Scale bars indicate $10 \mu \mathrm{m}$. (C) Fluorescence intensity profile of the cells in 
Figure 4B. Red curve indicated Lamin B1 channel and the blue curve indicated DAPI channel. (D) Quantification analysis demonstrated majority of neurons have abnormal lamin B1 distribution after ADP-2 treatment and illumination (+ADP-2 +UV). Three independent experiments were analyzed. More than 75 cells were counted and analyzed in each groups. ** indicate significant at $p<0.01$ (analyzed by student's t-test).

A
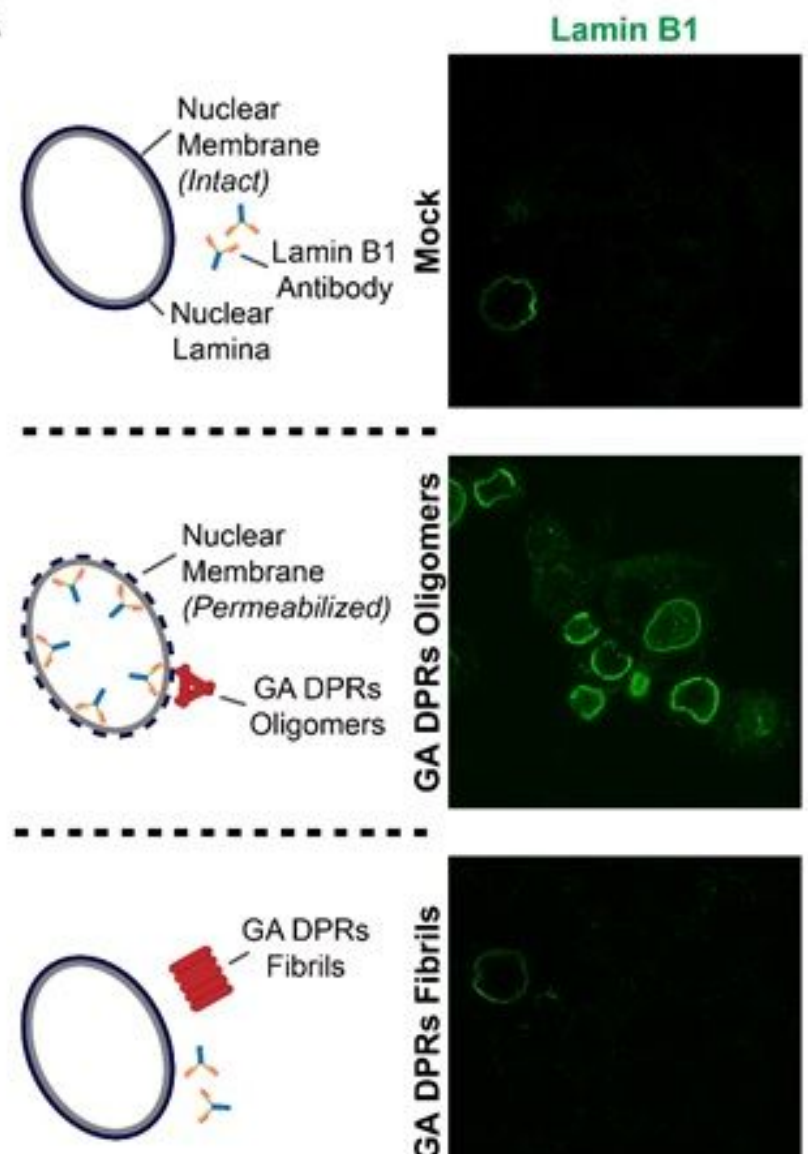

B
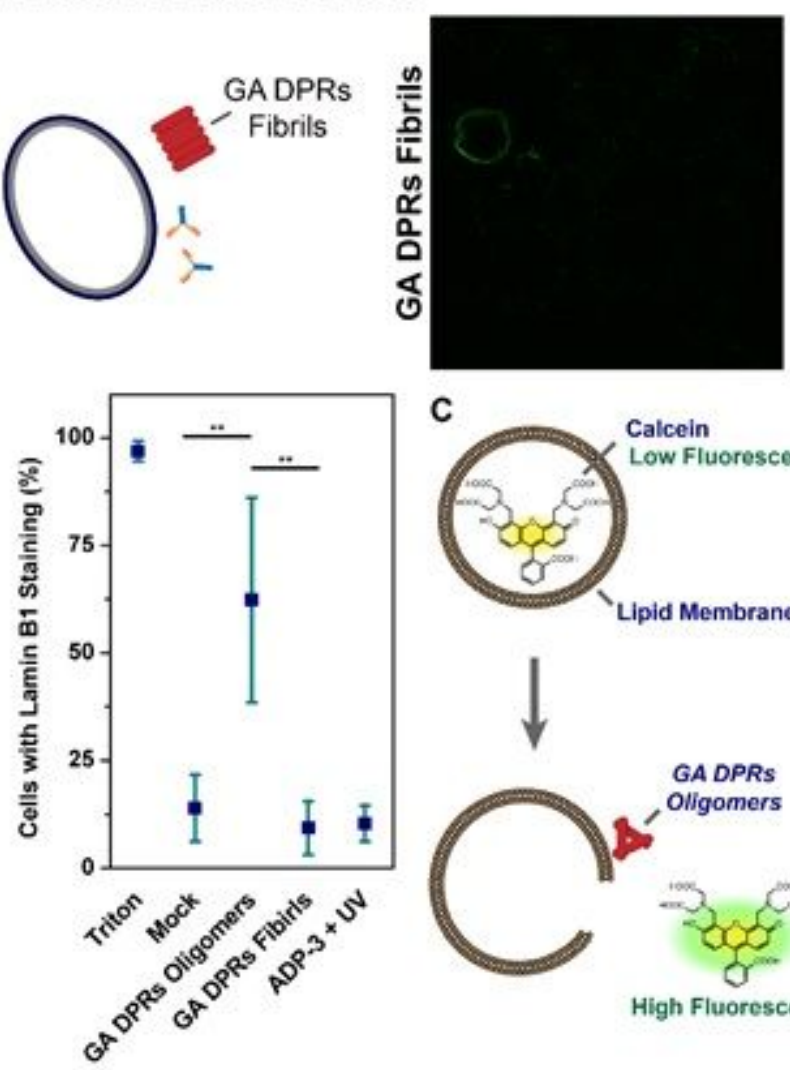

c

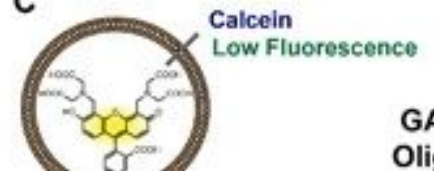

Lipid Membrane

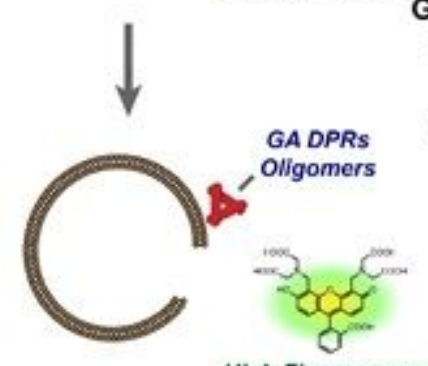

High Fluorescence

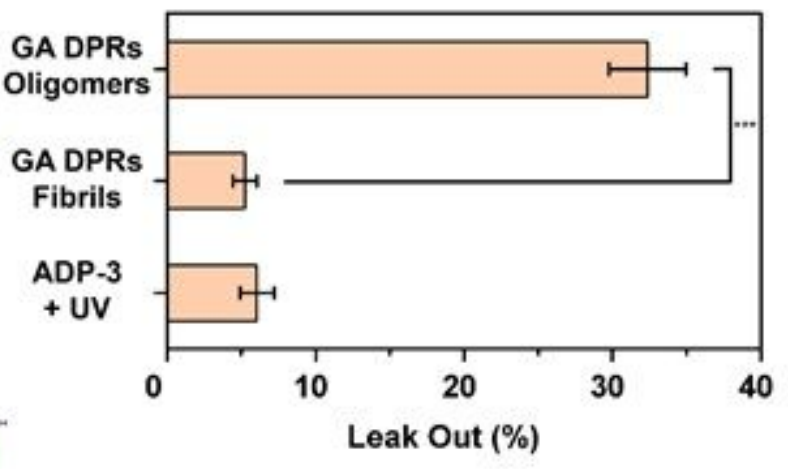

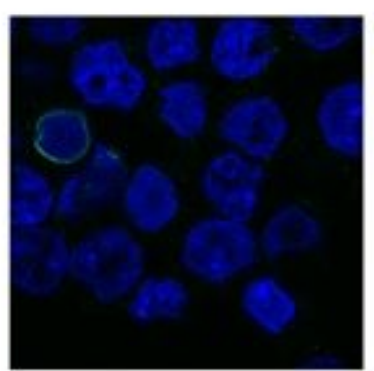
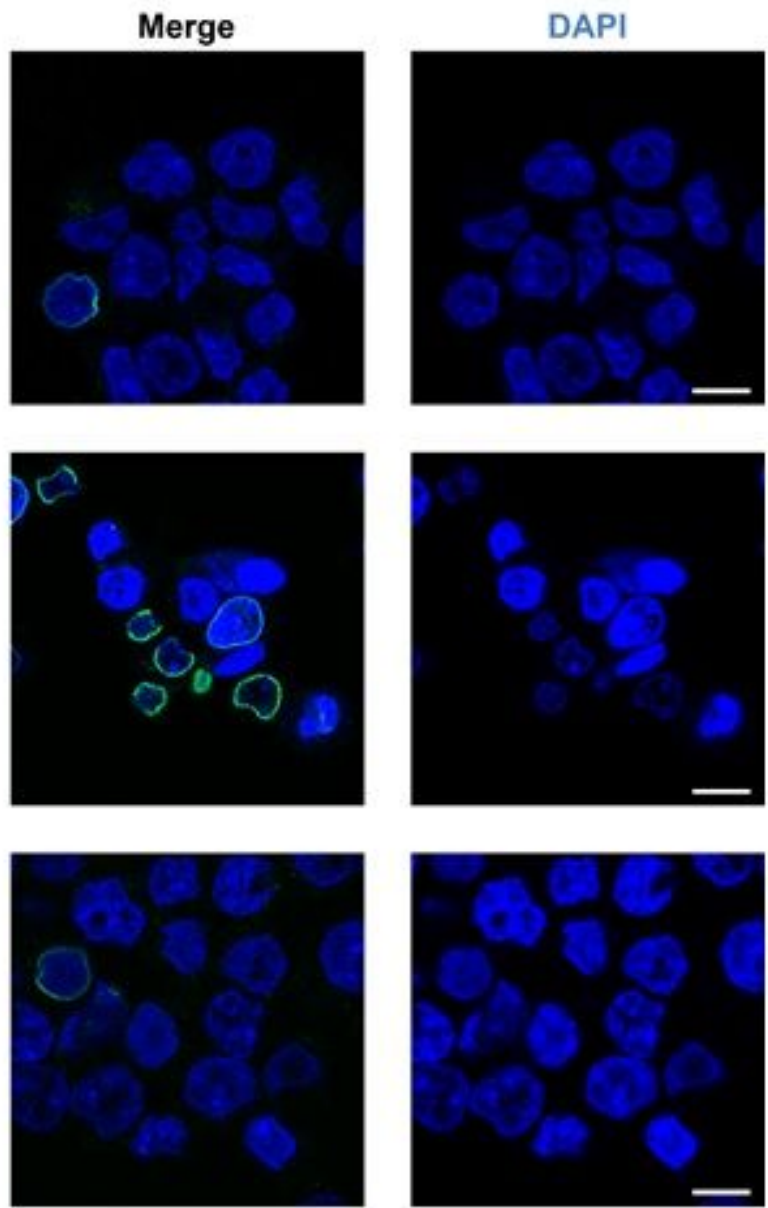

Figure 5 
GA DPRs oligomers permeablize the nuclear membrane. (A) Representative images of lamin B1 staining on digitonin-treated SH-SY5Y cells in the presence of GA DPRs oiligomers (upper row), fibrils (middle), or mock(buffer only, bottom row). The nuclei was counterstained with DAPI. Scale bars indicate $10 \mu \mathrm{m}$. (B) Quantification analysis of SH-SY5Y cell nuclei with lamin B1 staining. Four independent experiments were analyzed. More than 200 nuclei were counted and analyzed in each groups. $* \star$ indicate significant $p$ $<0.01$ (analyzed by student's t-test). (C) Ratio of the calcein (emission at $520 \mathrm{~nm}$ ) leak-out from lipidbased liposome based on the fluorescence measurement. Fluorescence intensity was measured for each sample, and then subtracts the background (liposome only) and normalized to the signal from the Triton X100 treatment (lysed liposome). *** indicated significant, $\mathrm{p}<0.001$ (analyzed by student's t-test).
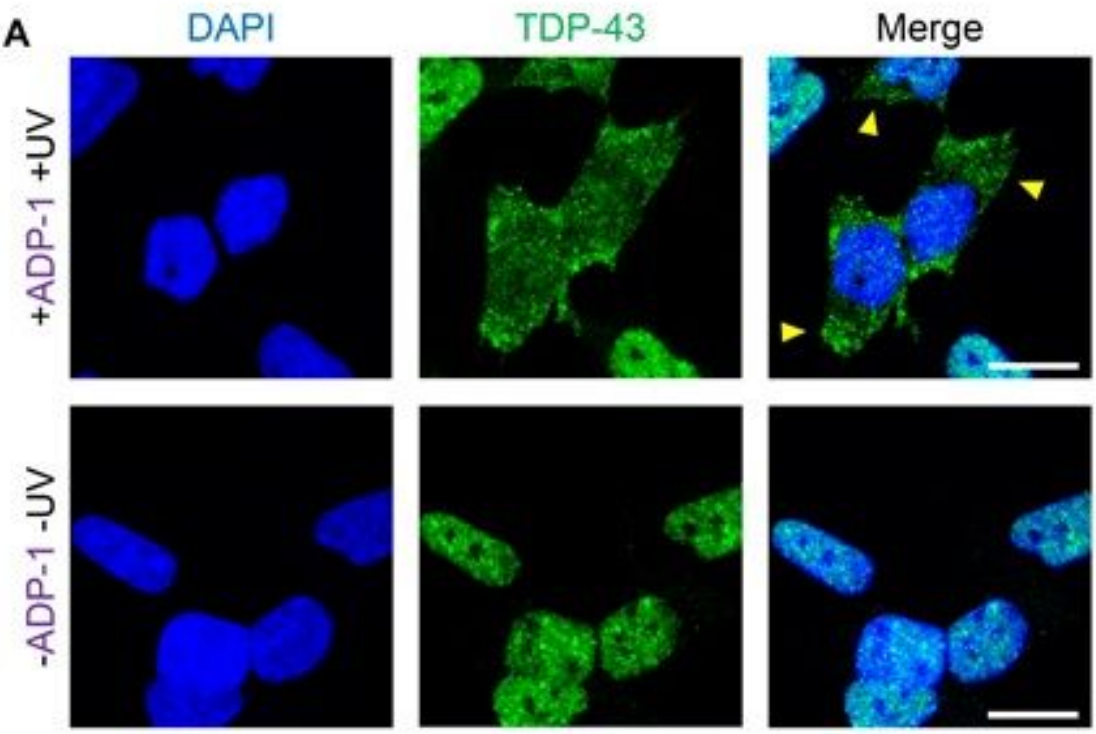

C
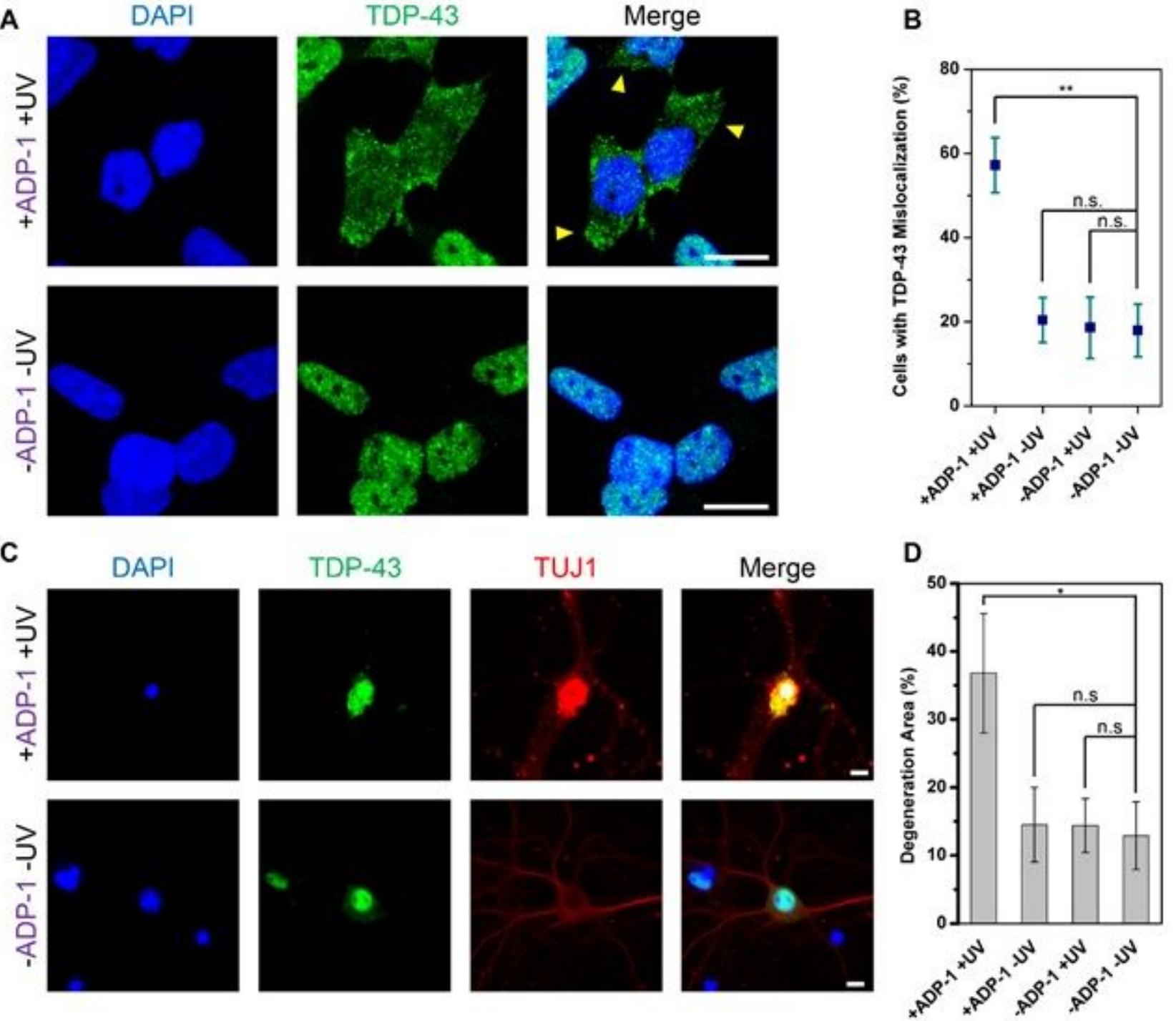

\section{Figure 6}

GA DPRs induces TDP-43 mislocalization and causes severe damage to the mouse cortical neurons. (A) Representive immunofluorescence of endogenous TDP-43 (Green) in neuroblastoma SH-SY5Y after ADP1 treatment $(1 \mu \mathrm{M})$ and photoinitiation. Yellow arrows indicate the cells with TDP-43 mislocalization. 
Scale bars indicate $10 \mu \mathrm{m}$. (B) Quantification analysis of SH-SY5Y cells with TDP-43 mislocalization. Three independent experiments were analyzed. More than 30 neurons were counted and analyzed in each groups. n.s. indicated not significant; ${ }^{* \star}$ indicate significant, $p<0.01$ (analyzed by one-way ANOVA). (C) Representative immunofluorescence images of 22DIV mouse cortical neurons stained with antibody against $\beta$-III-tubulin (red) and TDP-43 (green) treated with or without $1 \mu$ M ADP-1 and/or 2 minutes of UV irradiation. The DNA counter stain DAPI (blue) was included to identify the location of the nucleus. Scale bar indicated $10 \mu \mathrm{m}$. (D) Quantification analysis of degeneration area in 22DIV mouse cortical neurons. Three independent experiments were analyzed. More than 45 neurons were counted and analyzed in each groups. n.s. indicated not significant; * indicate significant $p<0.05$ (analyzed by one-way ANOVA).

\section{Supplementary Files}

This is a list of supplementary files associated with this preprint. Click to download.

- SupplementaryInformation.docx 